\title{
ARITHMETIC TORELLI MAPS FOR CUBIC SURFACES AND THREEFOLDS
}

\author{
JEFFREY D. ACHTER
}

\begin{abstract}
It has long been known that to a complex cubic surface or threefold one can canonically associate a principally polarized abelian variety. We give a construction which works for cubics over an arithmetic base, and in particular identifies the moduli space of cubic surfaces with an open substack of a certain moduli space of abelian varieties. This answers, away from the prime 2, an old question of Deligne and a recent question of Kudla and Rapoport.
\end{abstract}

\section{INTRODUCTION}

Consider a complex cubic surface. By associating to it either a K3 surface [19] or a cubic threefold [3, 4], one can construct a polarized Hodge structure. The Hodge structures which arise are parametrized by $\mathbb{B}^{4}$, the complex 4-ball, and in this way one can show that the moduli space $\mathcal{S}_{\mathrm{C}}$ of complex cubic surfaces is uniformized by $\mathbb{B}^{4}$.

It turns out that the relevant arithmetic quotient of $\mathbb{B}^{4}$ is the set of complex points of $\mathcal{M}$, the moduli space of abelian fivefolds with action by $\mathbb{Z}\left[\zeta_{3}\right]$ of signature $(4,1)$, and there is a Torelli map $\tau_{\mathbb{C}}: \mathcal{S}_{\mathbb{C}} \rightarrow \mathcal{M}_{\mathbb{C}}$. The main goal of the present paper is to construct an arithmetic Torelli map for cubic surfaces:

Theorem A. There is an open immersion

$$
\mathcal{S} \longrightarrow \mathcal{M}
$$

of stacks over $\mathbb{Z}\left[\zeta_{3}, 1 / 6\right]$ which specializes to $\tau_{\mathrm{C}}$.

We refer to Proposition 4.5(b) and Theorem 5.7 for more details. In particular, this immersion extends to a homeomorphism if $\mathcal{S}$ is replaced with its partial compactification $\mathcal{S}_{\text {st }}$, the moduli stack of stable cubic surfaces. Theorem Ashows that $\mathcal{S}_{\text {st }}$ is essentially a Shimura variety, and thus supports unexpected arithmetic structures, such as Hecke correspondences and modular forms.

It turns out that a key step in understanding the Torelli map for cubic surfaces is to show that there is an arithmetic construction which recovers the intermediate Jacobian of a complex cubic threefold. Let $\widetilde{\mathcal{T}} \subset$

2010 Mathematics Subject Classification. Primary 14J10; Secondary 11G18, 14H40, 14K30.

This work was partially supported by a grant from the Simons foundation (204164). 
$H^{0}\left(\mathbb{P}^{4}, \mathcal{O}_{\mathbb{P}^{4}}(3)\right)$ be the scheme of smooth cubic forms in five variables, and let $\jmath_{\mathrm{C}}: \widetilde{\mathcal{T}}_{\mathrm{C}} \rightarrow \mathcal{A}_{5, \mathrm{C}}$ be the intermediate Jacobian functor.

Theorem B. There is a morphism

$$
\widetilde{\mathcal{T}} \longrightarrow \mathcal{A}_{5}
$$

of stacks over $\mathbb{Z}[1 / 2]$ which specializes to $]_{C}$.

(See Theorem 3.4 and Corollary 3.5 below.)

Both of these results are statements about arithmetic period maps, but with slightly different antecedents. Theorems $B$ and $\mathrm{A}$ provide special cases of conjectures of, respectively, Deligne on algebraic period maps and of Kudla and Rapoport on occult period maps.

1.1. Algebraic period maps. It is a classical insight that one can study a complex variety by examining its periods. For a Riemann surface, the cokernel of the period lattice is not just a complex analytic torus but an algebraizable one. Constructing this abelian variety through algebro-geometric, as opposed to transcendental, means was a central concern of abstract algebraic geometry. More recently, although still classically, one understood how to make sense of this construction in families. (See [23] for a comprehensive survey.)

Similarly, Clemens and Griffiths [15] show that a complex cubic threefold is characterized by its middle cohomology. The intermediate Jacobian of such a variety is a principally polarized abelian fivefold, and Clemens and Griffiths prove a global Torelli theorem. Mumford gives an algebrogeometric construction (via Prym varieties) of the intermediate Jacobian, and thus reveals the essential algebraic nature of this a priori transcendental object. Beauville and Murre extend these ideas to cubic threefolds over algebraically closed fields of (almost) arbitrary characteristic. Theorem B states that these constructions make sense not just pointwise but for families over a general base. Thus, it provides a new example of an algebraic period map. (In fairness, one should note that the essential algebraization step is provided by the existence of Picard schemes of relative curves.)

In [16], Deligne studies the period map, via intermediate Jacobians, for smooth complete intersections of Hodge level one. He proves that the universal intermediate Jacobian attached to the complex fiber descends to $\mathbb{Q}$, and conjectures that it spread out to $\mathbb{Z}$. Theorem $\mathbb{B}$ is apparently the first nontrivial case of Deligne's conjecture (away from the prime 2). In joint work with Casalaina-Martin, the author is currently investigating the extent to which the techniques of the present work allow the resolution of other cases of Deligne's conjecture. (The key point is that a cubic threefold is, after suitable blowup, a quadric fibration; examination of the list [41] of complete intersections of Hodge level one indicates that similar progress may be made in other cases.) 
1.2. Occult period maps. The moduli space of stable complex cubic surfaces is an arithmetic quotient of the complex 4-ball. Thanks to work of, e.g., Allcock, Carlson and Toledo [5], Looijenga and Swierstra [27], Kondō and Dolgachev and Kondō [20, 24], several other examples are known of apparently accidental isomorphisms between certain moduli spaces of complex varieties and (open subsets of) arithmetic quotients of complex unit balls. As part of their work on unitary Shimura varieties [25], Kudla and Rapoport survey such occult period maps ("occult", because their construction relies not on the periods of the varieties in question, but on other, auxiliary varieties) [26], and show that they descend to certain cyclotomic number fields $\mathbb{Q}\left(\zeta_{n}\right)$. They conjecture [25, Conj. 15.9] that each such descends to $\mathbb{Z}\left[\zeta_{n}, 1 / n\right]$. Thus (away from 2), Theorem $\mathrm{A}$ secures one case of the conjecture of Kudla and Rapoport. The construction given here depends on the fact that one knows how to construct the Picard scheme of a relative curve; the algebraicity of $\tau$ comes from that of the classical Torelli map for curves.

1.3. Alternate approaches. Like [4], the present work constructs the occult period map $\tau$ by associating, to each cubic surface, the cyclic cubic threefold ramified along that surface, and then constructing an associated abelian variety.

There are other methods available for demonstrating an exceptional isomorphism between complex moduli spaces and ball quotients, and they may well yield novel approaches to and extensions of Theorem A.

In [17], the authors show that certain moduli spaces of weighted points in $\mathbb{P}^{1}$ are ball quotients, by using the period map for cyclic covers of the projective line. Some of these results have been recovered by instead considering periods of K3 surfaces. Many of the occult period maps reviewed in [26], and indeed Dolgachev, van Geemen and Kondō's construction of $\tau_{\mathbb{C}}$, go through a construction involving K3 surfaces. Recent work on arithmetic period maps for K3 surfaces may well allow progress on the problem of arithmetic occult periods; the author hopes to return to this subject shortly.

1.4. Outline. After establishing notation in Section 2 in Section 3 we construct a period morphism $\widetilde{\omega}: \widetilde{\mathcal{T}} \rightarrow \mathcal{A}_{5}$ from the space of smooth cubic forms in five variables to the moduli space of abelian fivefolds. This is used in Section 4 to define the morphism of $\mathbb{Z}\left[\zeta_{3}, 1 / 6\right]$-stacks $\tau: \mathcal{S} \rightarrow \mathcal{M}$. (The reader is invited to consult diagram 4.2.1 to see how these morphisms fit together.) In Section 5, we characterize the image of $\tau$ and ultimately extend the domain of $\tau$ to the moduli space of semistable cubic surfaces.

The existence of an open immersion $\mathcal{S} \hookrightarrow \mathcal{M}$ over $\mathbb{Z}\left[\zeta_{3}, 1 / 6\right]$ means that information about the arithmetic and geometry of abelian varieties can be transported to the setting of cubic surfaces. In a companion work [1], the author exploits this connection in order to classify the abelian varieties 
which arise as intermediate Jacobians of cubic threefolds attached to cubic surfaces.

Acknowledgments. It's a pleasure to thank, especially, M. Rapoport and D. Toledo for their encouragement; S. Casalaina-Martin for helpful conversations, particularly concerning the boundary of the moduli space of cubic threefolds; and the referee for insightful suggestions.

\section{MODUli SPACES}

Let $\mathcal{O}_{E}$ be the ring of Eisenstein integers $\mathbb{Z}\left[\zeta_{3}\right]$, and let $E=\mathbb{Q}\left(\zeta_{3}\right)$. In Sections 2.1 and 3 , all constructions are over $\mathbb{Z}[1 / 2]$; elsewhere, all spaces are objects over $\mathcal{O}_{E}[1 / 6]$. The present section establishes notation concerning moduli spaces of cubic surfaces and threefolds (2.1) and abelian varieties (2.2).

2.1. Cubics. Let $\widetilde{\mathcal{S}} \subset H^{0}\left(\mathbb{P}^{3}, \mathcal{O}_{\mathbb{P}^{3}}(3)\right) \rightarrow$ Spec $\mathbb{Z}$ be the space of smooth cubic forms in four variables. Similarly, let $\widetilde{\mathcal{S}}_{\text {st }}$ and $\widetilde{\mathcal{S}}_{\text {ss }}$ be the spaces of stable and semistable cubic forms, respectively, in the sense of GIT [34, Sec. 4.2]. The complement $\widetilde{\mathcal{S}}_{\text {st }} \backslash \widetilde{\mathcal{S}}$ is a horizontal divisor, i.e., for each $t \in \operatorname{Spec} \mathbb{Z}$, the fiber $\left(\widetilde{\mathcal{S}}_{\text {st }} \backslash \widetilde{\mathcal{S}}\right)_{t}$ has codimension one in $\widetilde{\mathcal{S}}_{\text {st }}$. Moreover, $\widetilde{\mathcal{S}}_{\text {ss }} \backslash \widetilde{\mathcal{S}}_{\text {st }}$ forms a single orbit under the action of $\mathrm{SL}_{4}$.

Let $\mathcal{S}=\widetilde{\mathcal{S}} / \mathrm{SL}_{4}$ be the moduli stack of smooth projective cubic surfaces, and similarly define $\mathcal{S}_{\text {st }}$ and $\mathcal{S}_{\text {ss }}$. A cubic surface is stable if it is either smooth or has ordinary double points of type $A_{1}$ [33, 1.14].

The coarse moduli space $\underline{S}_{\mathrm{ss}}$ of $\mathcal{S}_{\mathrm{ss}}$ is a normal projective scheme. Let $g: \mathcal{Y} \rightarrow \mathcal{S}$ be the tautological cubic surface over $\mathcal{S}$. We will slightly abuse notation and also let $\mathcal{Y} \rightarrow \widetilde{\mathcal{S}}$ be the universal cubic surface over $\widetilde{\mathcal{S}}$, thought of as a subscheme of $\mathbb{P}_{\widetilde{\mathcal{S}}}^{3}$.

We follow [28, Sec. 2] for the notion of marked cubic surface used here. More precisely, we give a definition of marked cubic surfaces which, in the special case of complex cubic surfaces, coincides with the definition in [28].

Recall that if $Y / K$ is a cubic surface over an algebraically closed field, then there are 27 lines on $Y$. Let $\Lambda(Y)$ be the simple graph with a vertex $[L]$ for each line $L$ on $Y$, where $[L]$ and $\left[L^{\prime}\right]$ are adjacent if and only if $L$ and $L^{\prime}$ have nontrivial intersection.

It is known that $\Lambda(Y)$ is isomorphic to the abstract graph $\Lambda_{0}$ defined as follows. For $1 \leq i \leq 6$, there are vertices $e_{i}$ and $c_{i}$, and for $1 \leq j<k \leq 6$ there is a vertex $\ell_{j k}$. Then $e_{i}$ and $c_{j}$ are adjacent if $i \neq j$; each of $e_{i}$ and $c_{i}$ is adjacent to $\ell_{j k}$ if $i \in\{j, k\} ; \ell_{i j}$ and $\ell_{k m}$ are adjacent if $\{i, j\} \cap\{k, m\}=\varnothing$; and these are the only adjacencies in $\Lambda_{0}$.

A marking of $Y$ is a graph isomorphism $\Psi: \Lambda(Y) \rightarrow \Lambda_{0}$. We briefly indicate how such a marking may be constructed. Realize $Y$ as the blowup $Y \rightarrow \mathbb{P}^{2}$ of the projective plane at 6 points $P_{1}, \cdots, P_{6}$. Let $E_{i}$ be the inverse image of $P_{i}$; for $1 \leq i<j \leq 6$ let $L_{i j}$ be the strict transform of the line 
connecting $P_{i}$ and $P_{j}$; and let $C_{i}$ be the strict transform of the conic through $\left\{P_{1}, \cdots, P_{6}\right\} \backslash P_{i}$. Then the rule $\left[E_{i}\right] \mapsto e_{i},\left[L_{i j}\right] \mapsto \ell_{i j},\left[C_{i}\right] \mapsto c_{i}$ is a marking of $Y$.

The automorphism group of $\Lambda_{0}$ is $\mathbb{W}=W\left(E_{6}\right)$, the Weyl group of the exceptional root system $\mathrm{E}_{6}$, and thus the set of markings on a fixed cubic surface is a torsor under $\mathbb{W}$. Moreover, an automorphism of a surface which fixes a marking is necessarily the identity [39. Prop. 1.1]. So, let $\mathcal{S}^{\mathrm{m}}$ be the moduli space of marked cubic surfaces $(Y, \Psi)$. Then $\mathcal{S}^{\mathrm{m}}$ is (represented by) a smooth quasiprojective scheme, and under the forgetful map we have $\mathcal{S}=\mathcal{S}^{\mathrm{m}} / \mathbb{W}$.

Let $\mathcal{S}_{\mathrm{st}}^{\mathrm{m}}$ be the normalization of $\mathcal{S}_{\mathrm{st}}$ in $\mathcal{S}^{\mathrm{m}}$, and similarly define $\mathcal{S}_{\mathrm{ss}}^{\mathrm{m}}$. (In [4], the complex fiber $\mathcal{S}_{\mathrm{st}, \mathbb{C}}^{\mathrm{m}}$ is constructed as a certain Fox completion; but these two notions coincide [17, 8.1].) Then $\mathcal{S}_{\mathrm{ss}}^{\mathrm{m}}$ is a normal projective scheme.

Finally, let $\widetilde{\mathcal{T}} \subset H^{0}\left(\mathbb{P}^{4}, \mathcal{O}_{\mathbb{P}^{4}}(3)\right)$ be the space of smooth cubic forms in five variables, and let $\mathcal{T}=\widetilde{\mathcal{T}} / \mathrm{SL}_{5}$ be the space of smooth projective cubic threefolds. Let $h: \mathcal{Z} \rightarrow \mathcal{T}$ denote both the tautological cubic threefold over $\mathcal{T}$ and its pullback to $\widetilde{\mathcal{T}}$. For the sake of completeness, we record the fact that a cubic threefold is stable if and only if each of its singularities has type $A_{j}$ with $j \leq 4$. (This is [2, Thm. 1.1]. While the result there is only claimed for complex threefolds, the proof relies only on the Hilbert-Mumford stability criterion [35, Thm. 2.1], which is valid in all characteristics [42, Thm. 2.2].)

Taken together, we have parameter spaces $\widetilde{\mathcal{S}}$ and $\widetilde{\mathcal{T}}$ for smooth cubic forms in four and five variables, respectively. Each of these is an open subscheme of a projective space over $\operatorname{Spec} \mathbb{Z}$, and in particular Noetherian and smooth. Taking GIT quotients, we have smooth Deligne-Mumford stacks $\mathcal{S}$ and $\mathcal{T}$ over Spec $\mathbb{Z}$. In the sequel we will consider these as objects over $\operatorname{Spec} \mathcal{O}_{E}[1 / 6]$ and Spec $\mathbb{Z}[1 / 2]$, respectively, and in particular set aside the question of how to mark a cubic surface in characteristic three.

2.2. Abelian varieties. We work in the category of $\mathcal{O}_{E}[1 / 6]$-schemes. In particular, each scheme $S$ comes equipped with a morphism $\iota: \mathcal{O}_{E} \rightarrow \mathcal{O}_{S}$. Let $\bar{l}: \mathcal{O}_{E} \rightarrow \mathcal{O}_{S}$ be the composition of the involution of $\mathcal{O}_{E}$ and the morphism $\iota$. Then $\mathcal{O}_{S} \otimes \mathcal{O}_{E} \cong \mathcal{O}_{S} \oplus \mathcal{O}_{S}$, where $\mathcal{O}_{E}$ acts on the first copy of $\mathcal{O}_{S}$ via $\iota$ and on the second by $\bar{l}$. Under this decomposition, any locally free sheaf $\mathcal{F}$ of $\mathcal{O}_{S} \otimes \mathcal{O}_{E}$-modules is a direct sum of two locally free $\mathcal{O}_{S}$-modules of ranks $r$ and $s$. The pair $(r, s)$ is called the signature of $\mathcal{F}$.

Let $\mathcal{M}=\mathcal{M}_{(4,1)}$ be the moduli stack of principally polarized abelian fivefolds equipped with an $\mathcal{O}_{E}$-action of signature $(4,1)$. More precisely, $\mathcal{M}(S)$ consists of triples $(X, l, \lambda)$, where $X \rightarrow S$ is an abelian scheme of relative dimension five; $\iota: \mathcal{O}_{E} \rightarrow \operatorname{End}_{S}(X)$ is an action of $\mathcal{O}_{E}$ such that $\operatorname{Lie}(X)$ has signature $(4,1)$; and $\lambda$ is a principal polarization compatible with the action of $\mathcal{O}_{E}$. For each prime $\ell$ invertible on $S$, the principal polarization $\lambda$ induces a skew-symmetric unimodular form on the $\ell$-adic Tate module, 
$\langle\cdot, \cdot\rangle_{\lambda}: T_{\ell} X \times T_{\ell} X \rightarrow \mathbb{Z}_{\ell}(1)$. The compatibility of $\lambda$ and $\iota$ may be expressed by insisting that for $x, y \in T_{\ell} X$ and $a \in \mathcal{O}_{E},\langle\iota(a) x, y\rangle_{\lambda}=\langle x, \bar{\imath}(a) y\rangle_{\lambda}$. The forgetful morphism $]: \mathcal{M} \rightarrow \mathcal{A}_{5}$ is an embedding, since an abelian fivefold admits at most one $\mathbb{Z}\left[\zeta_{3}\right]$-action of signature $(4,1)$. Let $f: \mathcal{X} \rightarrow \mathcal{A}_{5}$ denote both the tautological abelian scheme over $\mathcal{A}_{5}$ and its pullback via $\jmath$ to $\mathcal{M}$.

For our construction of the Torelli map, we will need the notion of a certain kind of level structure on an $\mathcal{O}_{E}$-abelian variety. For a rational prime $\ell$, let $\mathcal{O}_{E, \ell}=\mathcal{O}_{E} \otimes \mathbb{Z}_{\ell}$; then $\mathcal{O}_{E, \ell}$ is a degree two $\mathbb{Z}_{\ell}$-algebra with involution, and $T_{\ell} X$ is free of rank five over $\mathcal{O}_{E, \ell}$. From general theory (e.g., [25, 2.3]), there exists a unique unimodular $\mathcal{O}_{E, \ell}$-Hermitian form $h_{\lambda}: T_{\ell} X \times T_{\ell} X \rightarrow$ $\mathcal{O}_{E, \ell}(1)$ on $T_{\ell} X$ such that, for $x, y \in T_{\ell} X,\langle x, y\rangle_{\lambda}=\operatorname{tr}_{\mathcal{O}_{E, \ell} / \mathbb{Z}_{\ell}}\left(h_{\lambda}(x, y) / \sqrt{-3}\right)$.

Apply these considerations in the special case $\ell=3$, and reduce $h_{\lambda}$ modulo $\left(1-\zeta_{3}\right)$. Note that $\mathcal{O}_{E, 3} /\left(1-\zeta_{3}\right) \cong \mathcal{O}_{E} /\left(1-\zeta_{3}\right) \cong \mathbb{F}_{3}$; that $T_{\ell} X \otimes$ $\mathcal{O}_{E, \ell} /\left(1-\zeta_{3}\right)$ is a five-dimensional vector space over the residue field $\mathbb{F}_{3}$; and that the choice of $\zeta_{3}$ yields an isomorphism $\left(\mathcal{O}_{E, \ell} /\left(1-\zeta_{3}\right)\right)(1) \cong \mathbb{F}_{3}$. Suppose that $X\left[1-\zeta_{3}\right]$, the $\left(1-\zeta_{3}\right)$-torsion subscheme of $X$, is split over $S$, so that $X\left[1-\zeta_{3}\right](S) \cong \mathbb{F}_{3}^{\oplus 5}$. (For an arbitrary $\mathcal{O}_{E}$-abelian scheme, this will only happen after étale extension of the base.) Then $\lambda$ induces a perfect pairing

$$
X\left[1-\zeta_{3}\right](S) \times X\left[1-\zeta_{3}\right](S) \stackrel{\bar{h}_{\lambda}}{\longrightarrow} \mathbb{F}_{3} .
$$

Since the involution of $\mathcal{O}_{E}$ is trivial modulo $\left(1-\zeta_{3}\right)$, this Hermitian pairing is actually an orthogonal form on $X\left[1-\zeta_{3}\right](S)$.

Let $\left(V_{0}, q_{0}\right)$ be a five-dimensional vector space over $\mathbb{F}_{3}$ equipped with a perfect orthogonal form $q_{0}$. A $\left(1-\zeta_{3}\right)$-level structure on $(X, l, \lambda)$ is an isomorphism $\Phi:\left(V_{0}, q_{0}\right) \rightarrow\left(X\left[1-\zeta_{3}\right](S), \bar{h}_{\lambda}\right)$ of quadratic spaces; equivalently, it is a choice of basis on $X\left[1-\zeta_{3}\right](S)$ which is orthonormal for $\bar{h}_{\lambda}$.

Let $\mathcal{M}^{\left(1-\zeta_{3}\right)}$ be the moduli stack of principally polarized $\mathcal{O}_{E}$-abelian varieties with level $\left(1-\zeta_{3}\right)$ structure, i.e., of quadruples $(X, l, \lambda, \Phi)$. The usual Serre lemma shows that any automorphism of $(X, l, \lambda)$ which fixes $\Phi$ is necessarily the identity automorphism, so $\mathcal{M}^{\left(1-\zeta_{3}\right)}$ is a smooth quasiprojective scheme.

The orthogonal group $\mathrm{O}\left(V_{0}, q_{0}\right)$ acts on the set of $\left(1-\zeta_{3}\right)$-level structures on $(X, l, \lambda)$. Moreover, the center of $\mathrm{O}\left(V_{0}, q_{0}\right)$ acts trivially on such level structures (e.g., [28, Rem. 4.2]), so that the forgetful map $\mathcal{M}^{\left(1-\zeta_{3}\right)} \rightarrow \mathcal{M}$ has covering group $\mathrm{O}\left(V_{0}, q_{0}\right) /\{ \pm 1\}=\mathrm{PO}\left(V_{0}, q_{0}\right)$. From the description of $\mathbb{W}$ as the automorphism group of the Euclidean lattice $E_{6}$, one can extract an inclusion $\mathbb{W} \rightarrow \mathrm{O}\left(V_{0}, q_{0}\right)$ such that the composition $\mathbb{W} \hookrightarrow \mathrm{O}\left(V_{0}, q_{0}\right) \rightarrow$ $\mathrm{PO}\left(V_{0}, q_{0}\right)$ is an isomorphism.

Let $\mathcal{M}$ be the minimal (also known as the Satake, or Baily-Borel) compactification of $\mathcal{M}$, and let $\overline{\mathcal{M}}^{\left(1-\zeta_{3}\right)}$ be the normalization of $\overline{\mathcal{M}}$ in $\mathcal{M}^{\left(1-\zeta_{3}\right)}$. The coarse moduli space $\underline{\bar{M}}$ of $\overline{\mathcal{M}}$ is a normal projective scheme, and $\overline{\mathcal{M}}^{\left(1-\zeta_{3}\right)}$ is itself represented by a normal projective scheme. 
2.3. A remark on characteristic. As is hopefully clear from the introduction, much of the work of the present paper is to show that transcendental facts over $\mathbb{C}$ have their origins in algebraic facts over an arithmetic base.

In Section 3, we already find it necessary to invert the prime 2 in order to make an algebraic version of the "intermediate Jacobian" functor for cubic threefolds. Initially, this is because we make heavy use of results from [7] and [36] on quadric fibrations, especially those coming from cubic threefolds. These results are valid over an arbitrary algebraically closed field whose characteristic is not 2 . Even if one were able to circumvent this difficulty, it is still not clear that one can carry out the Prym construction in even characteristic. Poincaré reducibility is apparently not known for arbitrary abelian schemes. Thus, the invertibility of 2 is used in an essential way in Lemma 3.8 .

It is perhaps less surprising that, starting in Section 4 , we find it expedient to invert 3 . The initial construction starts with a certain cyclic cover of degree 3, and then proceeds through a comparison involving abelian schemes with $\mathbb{Z}\left[\zeta_{3}\right]$-action and level structure at 3 . Each of these notions makes sense in characteristic 3 , but is considerably more delicate.

\section{Cubic THREEFOLDS}

After recalling the Prym construction of the intermediate Jacobian of a cubic threefold over an algebraically closed field (Section 3.1), we use a descent argument to show that this construction works for a cubic threefold over an arbitrary field (Section 3.2), and even over an arbitrary normal Noetherian base scheme (Section 3.3). In particular, we deduce (Corollary 3.5) the existence of an arithmetic Torelli map $\omega: \widetilde{\mathcal{T}} \rightarrow \mathcal{A}_{5}$. As discussed below, this proves a special case of [16, 3.3]. The main construction of this section requires a theory of relative Prym schemes, which is worked out in Section 3.4

In this section, we work in the category of schemes over $\mathbb{Z}[1 / 2]$. In particular, we will only consider fields whose characteristic is either zero or odd.

3.1. Algebraically closed fields. Let $Z / \mathbb{C}$ be a cubic threefold. One can associate to $Z$ the intermediate Jacobian $J(Z)=\mathrm{F}^{2} H^{3}(Z, \mathbb{C}) \backslash H^{3}(Z, \mathbb{C}) / H^{3}(Z, \mathbb{Z})$, a principally polarized abelian fivefold. The map $\widetilde{\mathcal{T}}(\mathbb{C}) \rightarrow \mathcal{A}_{5}(\mathbb{C})$ comes from a complex analytic map $\widetilde{\mathcal{T}}_{\mathrm{C}} \rightarrow \mathcal{A}_{5, \mathrm{C}}$.

Alternatively, $J(Z)$ can be constructed without recourse to transcendental methods, using a Prym construction. Briefly, let $L \subset Z$ be a suitably generic line (in the sense of, say, [36, Prop. 1.25]). Projection to the space of planes through $L$ gives $Z^{*}$, the blowup of $Z$ along $L$, a structure of fibration $\omega_{L}: Z^{*} \rightarrow \mathbb{P}^{2}$. The fibers of $\omega_{L}$ are conics, which are smooth outside the discriminant locus $\Delta_{L} \subset \mathbb{P}^{2}$. Direct calculation (using the genericity of $L$ ) shows that $\Delta_{L}$ is a smooth, irreducible curve of genus 6 . Moreover, inside 
the Fano surface $F_{Z}$ of lines on $Z$ there is an étale double cover $\widetilde{\Delta}_{L}$ of $\Delta_{L}$, necessarily of genus 11 . There is a norm map $\mathcal{N}: \operatorname{Pic}^{0}\left(\widetilde{\Delta}_{L}\right) \rightarrow \operatorname{Pic}^{0}\left(\Delta_{L}\right)$ of principally polarized abelian varieties. Let $P_{L}$ be the connected component of identity of the kernel of $\mathcal{N}$. By Mumford's theory of the Prym variety, $P_{L}$ is an abelian variety which inherits a canonical principal polarization from that of $\operatorname{Pic}^{0}\left(\widetilde{\Delta}_{L}\right)$. It turns out that $J(Z)$ is isomorphic to $P_{L}$ as a principally polarized abelian variety.

The Prym construction is already well-understood over algebraically closed fields of any characteristic (other than two) [7, 36]. The goal of the present section is to show that this construction makes sense in families.

Thanks to Beauville, Mumford and Murre, one knows that Prym varieties over algebraically closed fields have a certain universal property. This property is briefly recalled here, following [7, Sec. 3.2].

Let $Z / k$ be a cubic threefold over an algebraically closed field in which 2 is invertible, and as above let $Z^{*}$ be the blowup of $Z$ along a good line $L$. Let $A^{2}(Z)$ be the group of rational equivalence classes of cycles on $Z$ of codimension two which are algebraically equivalent to zero, and define $A^{2}\left(Z^{*}\right)$ analogously.

There is an isomorphism of abstract groups $P_{L}(k) \stackrel{\sim}{\rightarrow} A^{2}\left(Z^{*}\right)$ [7], Thm. 3.1]. Inverting this map gives an isomorphism of abstract groups $\alpha_{L}^{\prime}(k)$ : $A^{2}\left(Z^{*}\right) \stackrel{\sim}{\rightarrow} P_{L}(k)$ which is regular, as follows. For a smooth variety $T / k$, declare that a (set-theoretic) map $a: T(k) \rightarrow A^{2}\left(Z^{*}\right)$ is algebraic if there exists a cycle $\xi$ on $Z^{*} \times T$ such that, for $t \in T(k), \xi_{t}=a(t)$. Then $\alpha_{L}^{\prime}(k)$ is regular in the sense that for any algebraic map $T(k) \rightarrow A^{2}\left(Z^{*}\right)$, the composition $T(k) \rightarrow A^{2}\left(Z^{*}\right) \rightarrow P_{L}(k)$ is induced by a morphism $T \rightarrow P_{L}$ of varieties. Following Beauville, we will abuse notation somewhat and write this morphism as $\alpha_{L}^{\prime}: A^{2}\left(Z^{*}\right) \rightarrow P_{L}$.

Beauville shows that $P_{L}$ is the universal algebraic representative of $A^{2}(Z)$ :

Proposition 3.1. $\quad$ (a) There is a regular map $\alpha_{L}: A^{2}(Z) \rightarrow P_{L}$ which induces an isomorphism $A^{2}(Z) \cong P_{L}(k)$.

(b) If $X / k$ is any abelian variety and $A^{2}(Z) \rightarrow X$ is regular, then there is a unique morphism $\beta_{X}: P_{L} \rightarrow X$ which makes the following diagram commute:

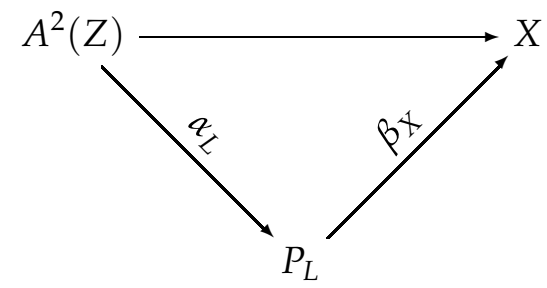

(c) There is a correspondence on $A^{2}(Z)$ which induces the principal polarization on $P_{L}$; in (3.1.1), if $A^{2}(Z) \rightarrow X$ is compatible with this correspondence, then $\beta_{X}$ is a morphism of polarized abelian varieties. 
Proof. There is a canonical isomorphism of abstract groups $A^{2}(Z) \cong A^{2}\left(Z^{*}\right)$ induced by the blowup morphism $b_{L}: Z^{*} \rightarrow Z$ [37, Lem. 2]. Suppose $T(k) \rightarrow A^{2}(Z)$ is algebraic, and represented by a cycle $\xi$ on $Z \times T$. Then the composition $T(k) \rightarrow A^{2}(Z) \stackrel{b_{L}^{*}}{\rightarrow} A^{2}\left(Z^{*}\right)$ is represented by the cycle $\left(b_{L}^{*} \times \mathrm{id}\right)(\xi)$, and thus algebraic. There is thus a regular map $\alpha_{L}: A^{2}(Z) \rightarrow$ $P_{L}$. Part (b) is precisely [7, Prop. 3.3]; $P_{L}$ is the unique algebraic representative of $A^{2}(Z)$. The compatibility with polarizations is [7, Prop. 3.5]. (We emphasize that the cited work of Beauville is valid over any algebraically closed field which is not of characteristic two.)

In particular, the principally polarized abelian variety $P_{L}$ is, up to canonical isomorphism, independent of the choice of (suitably generic) $L$, and we will denote this abelian variety by $P(Z)$.

3.2. Arbitrary fields. As a warmup we will show that, given a cubic threefold $Z$ over an arbitrary field $K$, one can canonically associate to it a principally polarized abelian fivefold over $K$.

Lemma 3.2. Let $Z / K$ be a cubic threefold.

(a) If $L$ is a sufficiently generic line on $Z_{\bar{K}}$, then $P_{L}\left(Z_{\bar{K}}\right)$ descends to $K$.

(b) If $M$ is a second sufficiently generic line on $Z_{\bar{K}}$, then there is a canonical isomorphism $P_{L}(Z) \rightarrow P_{M}(Z)$ of principally polarized abelian varieties over $K$.

Proof. Let $F=F_{Z} / K$ be the variety of lines on $Z$. Then $F$ is an irreducible smooth (Fano) surface (e.g., [36, 1.1], which is valid both in characteristic zero and in positive, odd characteristic). Let $F^{\text {good }} \subset F$ be the dense open subvariety constructed in [36. Prop. 1.25]; it is defined over K. Choose a finite Galois extension $K^{\prime}$ / $K$ such that there is a line $L$ (with moduli point) in $F^{\text {good }}\left(K^{\prime}\right)$. (Membership in $F^{\text {good }}$ is the "suitable genericity" referred to in Section 3.1.) The projection $\omega_{L}: Z_{K^{\prime}}^{*} \rightarrow \mathbb{P}^{2}$ is defined over $K^{\prime}$, and thus so is its discriminant locus $\Delta_{L} \subset \mathbb{P}^{2}$. In fact, $\Delta_{L}$ is a smooth, projective, absolutely irreducible curve of genus 6 .

Inside $F$, let $\widetilde{\Delta}_{L}$ be the set of (moduli points of) lines which meet $L$; then $\widetilde{\Delta}_{L}$ is a smooth, projective absolutely irreducible curve [36, Prop. 1.25.(iv)]. Since $L$ and $F$ are defined over $K^{\prime}$, so is $\widetilde{\Delta}_{L}$. Moreover, there is a natural étale double cover $\widetilde{\Delta}_{L} \rightarrow \Delta_{L}$; the fiber over a point of $\Delta_{L}$ consists of the two lines of the degenerate conic fiber over it. The Jacobians $\operatorname{Pic}^{0}\left(\widetilde{\Delta}_{L}\right)$ and $\operatorname{Pic}^{0}\left(\Delta_{L}\right)$ are also defined over $K^{\prime}$, and the Prym variety $P_{L}$ is a principally polarized abelian variety over $K^{\prime}$ (Lemma 3.8). We will use Galois descent (e.g., [11, Sec. 6.2.B] or [30, Thm. 6.23]) to show that these objects are actually defined over $K$.

Suppose $\sigma \in \operatorname{Gal}\left(K^{\prime} / K\right)$. Then $\sigma L:=L \times_{K^{\prime}, \sigma} K^{\prime}$ is also in $F^{\text {good }}\left(K^{\prime}\right)$, and $\sigma P_{L}$ may be calculated as $\sigma P_{L}=P_{\sigma L}$.

Let $N \geq 3$ be invertible in $K$; if necessary, enlarge $K^{\prime}$ so that $K^{\prime}$ is still Galois over $K$, but contains the field of definition of all $N$-torsion of each 
$P_{\sigma L}$. (Note that this does not enlarge the set of conjugates of $P_{L}$ under $\operatorname{Gal}\left(K^{\mathrm{sep}} / K\right)$.)

The universal property of Prym varieties (Proposition 3.1) implies that, for $\sigma \in \operatorname{Gal}\left(K^{\prime} / K\right)$, there is a canonical isomorphism $\beta_{\sigma, \bar{K}}: P_{\sigma L, \bar{K}} \stackrel{\sim}{\rightarrow} P_{L, \bar{K}}$ of principally polarized abelian varieties over $\bar{K}$ compatible with the maps $\alpha_{\sigma L, \bar{K}}: A^{2}\left(Z_{\bar{K}}\right) \rightarrow P_{\sigma L, \bar{K}}$ and $\alpha_{L, \bar{K}}: A^{2}\left(Z_{\bar{K}}\right) \rightarrow P_{L, \bar{K}}$. Since $K^{\prime}$ includes the field of definition of the $N$-torsion of each $P_{\sigma L}$, the isomorphism $\beta_{\sigma, \bar{K}}$ is actually defined over $K^{\prime}$ [44, Thm. 2.4]. Moreover, because $P_{\sigma L}=\sigma P_{L}$, we obtain canonical isomorphisms $\beta_{\sigma}: \sigma P_{L} \stackrel{\sim}{\rightarrow} P_{L}$. In particular, if $\sigma, \tau \in \operatorname{Gal}\left(K^{\prime} / K\right)$, then (again by Proposition 3.1) the following diagram commutes:

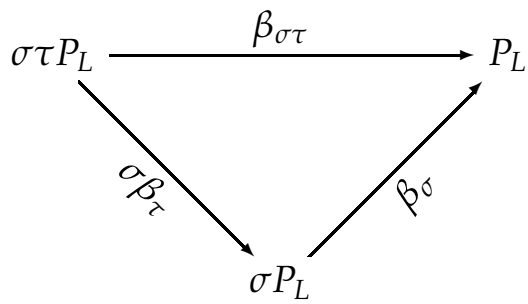

Therefore, the data $\left\{\beta_{\sigma}: \sigma \in \operatorname{Gal}\left(K^{\prime} / K\right)\right\}$ defines a $K^{\prime} / K$ descent datum on $P_{L}$. Because $P_{L}$ is projective, this descent datum is effective, and $P_{L}$ is defined over $K$. This proves (a).

The second part follows again from Galois descent. As above, let $K^{\prime}$ be a finite, Galois extension of $K$ such that $L, M$ and the $N$-torsion of the associated Pryms are all defined over $K^{\prime}$. Then for each $\sigma \in \operatorname{Gal}\left(K^{\prime} / K\right)$ there is a canonical isomorphism $P_{\sigma L}\left(Z_{\bar{K}}\right) \stackrel{\sim}{\rightarrow} P_{\sigma M}\left(Z_{\bar{K}}\right)$; this system descends to the desired isomorphism $P_{L}(Z) \stackrel{\sim}{\rightarrow} P_{M}(Z)$.

Consequently, if $Z / K$ is a cubic threefold over a field, one may canonically associate to it a principally polarized abelian fivefold, which will be simply denoted $P(Z)$.

Remark 3.3. Murre has shown [38, Thm. 7] that $P\left(Z_{\bar{K}}\right)$ is isomorphic to the Albanese variety of $F_{Z_{\bar{K}}}$. This, combined with the general fact that an Albanese variety exists over an arbitrary base field, gives an alternative proof of Lemma 3.2. However, the techniques used here will be deployed again over a more general base scheme in Theorem 3.4, while relative Albanese schemes are not known to exist in full generality.

3.3. Arbitrary base. In fact, the Prym construction makes sense for a cubic threefold over an arbitrary normal Noetherian base.

Theorem 3.4. Let $S$ be a normal Noetherian scheme over $\mathbb{Z}[1 / 2]$, and let $Z \rightarrow S$ be a relative smooth cubic threefold. There is a principally polarized abelian scheme $P(Z) \rightarrow S$ such that, for each point $s \in S, P(Z)_{s} \cong P\left(Z_{s}\right)$.

Proof. By definition, $Z$ comes equipped with an embedding inside a $\mathbb{P}^{4}$ bundle $\mathbb{P} V$ over $S$. Because $S$ is locally Noetherian we may consider $F \rightarrow S$, 
the scheme of lines on $Z$ [6, Thm. 3.3]. It is a smooth relative surface over $S$.

Let $F^{\text {good }} \subset F$ be the subscheme such that, for $s \in S,\left(F^{\text {good }}\right) s \cong F_{Z_{s}}^{\text {good }}$, where the latter is the nonempty, open subvariety constructed in [36, Prop. 1.25] and used in Lemma 3.2 .

Not only is every fiber of $F^{\text {good }} \rightarrow S$ dense in the corresponding fiber of $F \rightarrow S$, but $F^{\text {good }}$ is actually open in $F$. We briefly sketch why this is so. It suffices to verify the claim in the case where $S=\operatorname{Spec} A$ and $\mathbb{P} V \cong \mathbb{P}_{S}^{4}$. For a given point $s \in S$, Murre characterizes $F_{Z_{s}}^{\text {good }}$ by first constructing certain open subsets $U_{1, s}$ and $U_{2, s}$, given as the complement of the vanishing locus of certain explicit equations [36, eq. (11) and Lemma 1.11] involving a cubic form defining $Z_{s}$; then imposing an open condition $U_{3, s}$, that a smooth hyperplane section of $Z_{s}$ passes through the line in question; and then realizing $F_{Z_{s}}^{\text {good }}$ as the intersection of $U_{1, s}, U_{2, s}$ and $U_{3, s}$. In fact, for each $i \in\{1,2,3\}$, there is an open $U_{i} \subset F^{\text {good }}$ whose fiber at $s$ is $U_{i, s}$. For $i=3$, the Bertini-type condition is clearly open, while for $i=1,2$, one can simply use Murre's equations, now with coefficients in $A$.

Initially, suppose that there is a section of $F^{\text {good }} \rightarrow S$, corresponding to a relative line $L \subset Z \rightarrow S$. Let $G_{L} \rightarrow S$ be the Grassmann scheme of relative two-planes in $\mathbb{P} V$ which contain $L$; note that for $s \in S,\left(G_{L}\right)_{s} \cong \mathbb{P}_{s}^{2}$. Let $\Delta_{L} \subset G_{L}$ be the (discriminant) subscheme of planes which meet $Z$ in a union of three lines. For $s \in S,\left(\Delta_{L}\right)_{S}=\Delta_{L_{s}}$, and thus $\Delta_{L} \rightarrow S$ is a proper smooth irreducible relative curve of genus 6 . Similarly, let $\widetilde{\Delta}_{L} \subset F$ be the subscheme of lines which meet $L$. Then $\widetilde{\Delta}_{L} \rightarrow \Delta_{L}$ is an étale morphism of degree two, and $\widetilde{\Delta}_{L} \rightarrow S$ is a proper smooth irreducible relative curve of genus 11. By Lemma 3.8, there is a principally polarized complement $P(Z)$ for $\operatorname{Pic}^{0}\left(\Delta_{L} / S\right)$ inside $\operatorname{Pic}^{0}\left(\widetilde{\Delta}_{L} / S\right) ; P(Z)$ is the sought-for abelian scheme over $S$.

Now suppose that $F^{\text {good }} \rightarrow S$ does not admit a section. Since $S$ is normal, every connected component of $S$ is irreducible, and we may assume that $S$ itself is irreducible. Since $F^{\text {good }} \rightarrow S$ is smooth, it admits a section after étale base change. So, let $T \rightarrow S$ be an étale Galois morphism for which there exists a section $L \in F^{\text {good }}(T)$, corresponding to a line in $\mathbb{P} V$ contained in $Z_{T}$. The principally polarized abelian scheme $P_{L} \rightarrow T$ has already been constructed.

The relevant descent argument is now entirely similar to that used in Lemma 3.2. Let $\eta$ be the (disjoint) union of the generic points of $T$; note that each of these points is isomorphic to every other. By Lemma 3.2(b), for each $\sigma \in \operatorname{Aut}(T / S)$ there is a canonical isomorphism $P_{\sigma L, \eta} \stackrel{\sim}{\rightarrow} P_{L, \eta}$. Since $T$ is normal, each of these extends to an isomorphism $P_{\sigma L} \stackrel{\sim}{\rightarrow} P_{L}$ [21, Prop. I.2.7]. By Galois descent [11, Sec. 6.2.B], $P_{L}$ descends to $S$. 
Corollary 3.5. There is a morphism $\widetilde{\boldsymbol{\omega}}: \widetilde{\mathcal{T}} \rightarrow \mathcal{A}_{5}$ such that, for $t \in \widetilde{\mathcal{T}}, \mathcal{X}_{\widetilde{\omega}(t)} \cong$ $P\left(\mathcal{Z}_{s}\right)$.

Proof. The parameter space $\widetilde{\mathcal{T}}$ is itself represented by a normal Noetherian scheme; now use Theorem 3.4

Deligne has conjectured [16, 3.3] the existence of such an arithmetic Torelli map for any universal smooth complete intersection of Hodge level one. In fact, he proves that the universal intermediate Jacobian attached to the complex fiber descends to $\mathbb{Q}$, and conjectures that it spreads out to $\mathbb{Z}$. Corollary 3.5 proves this conjecture for the universal family of cubic threefolds, at least away from the prime 2. With this fact supplied, work of Deligne now gives a way to calculate the middle cohomology of the tautological cubic threefold:

Proposition 3.6. Let $\mathcal{P}=\widetilde{\mathcal{O}}^{*} \mathcal{X}$ be the relative Prym variety over $\widetilde{\mathcal{T}}$, with structural map $j: \mathcal{P} \rightarrow \widetilde{\mathcal{T}}$. Recall that $h: \mathcal{Z} \rightarrow \widetilde{\mathcal{T}}$ is the tautological smooth cubic threefold.

(a) For each rational prime $\ell$, there is an isomorphism of sheaves $R^{3} h_{*} \mathbb{Z}_{\ell}(1) \stackrel{\sim}{\rightarrow}$ $R^{1} j_{*} \mathbb{Z}_{\ell}$ on $\widetilde{\mathcal{T}}_{\mathbb{Z}[1 / 2 \ell]}$ compatible with the intersection forms;

(b) the canonical isomorphism $H_{\mathrm{dR}}^{3}\left(\mathcal{Z} / \widetilde{\mathcal{T}}_{\mathbb{C}}\right) \stackrel{\sim}{\rightarrow} H_{\mathrm{dR}}^{1}\left(\mathcal{P} / \widetilde{\mathcal{T}}_{\mathbb{C}}\right)$ of vector bundles with connection descends to $H_{\mathrm{dR}}^{3}\left(\mathcal{Z} / \widetilde{\mathcal{T}}_{\mathrm{Q}}\right) \stackrel{\sim}{\rightarrow} H_{\mathrm{dR}}^{1}\left(\mathcal{P} / \widetilde{\mathcal{T}}_{\mathrm{Q}}\right)$; and

(c) for each odd prime $p$, there is a canonical isomorphism $H_{\text {cris }}^{3}\left(\mathcal{Z} / \widetilde{\mathcal{T}}_{\mathbb{F}_{p}}\right) \stackrel{\sim}{\rightarrow}$ $H_{\text {cris }}^{1}\left(\mathcal{P} / \widetilde{\mathcal{T}}_{\mathbb{F}_{p}}\right)$ of isocrystals on $\widetilde{\mathcal{T}}_{\mathbb{F}_{p}}$.

Proof. We specialize the arguments of Deligne, which are valid for any universal smooth complete intersection of level one, to the case of cubic threefolds.

Part (a) is [16, Prop. 3.4].

For part (b), the canonical isomorphism of vector bundles on $\widetilde{\mathcal{T}}_{\mathrm{C}}$ may be described as follows. The transcendental construction of the intermediate Jacobian yields an isomorphism

$$
R^{3} h_{*} \mathbb{Z}(1) \stackrel{\sim}{\longrightarrow} R^{1} j_{*} \mathbb{Z}
$$

of polarized variations of Hodge structure on $\widetilde{\mathcal{T}}_{\mathbb{C}}$. Tensoring with the structure sheaf gives an isomorphism

$$
H_{\mathrm{dR}}^{3}\left(\mathcal{Z} / \widetilde{\mathcal{T}}_{\mathrm{C}}\right) \stackrel{\alpha_{\mathrm{C}}}{\longrightarrow} H_{\mathrm{dR}}^{1}\left(\mathcal{P} / \widetilde{\mathcal{T}}_{\mathrm{C}}\right)
$$

This isomorphism is compatible with the Gauss-Manin connection, and takes the cup product on $H_{\mathrm{dR}}^{3}\left(\mathcal{Z} / \widetilde{\mathcal{T}}_{\mathrm{C}}\right)$ to the polarization form on $H_{\mathrm{dR}}^{1}\left(\mathcal{P} / \widetilde{\mathcal{T}}_{\mathrm{C}}\right)$. Abstractly, one knows that there is some horizontal isomorphism $\beta: H_{\mathrm{dR}}^{3}\left(\mathcal{Z} / \widetilde{\mathcal{T}}_{\mathrm{Q}}\right) \stackrel{\sim}{\rightarrow}$ $H_{\mathrm{dR}}^{1}\left(\mathcal{P} / \widetilde{\mathcal{T}}_{\mathrm{Q}}\right)$ of vector bundles on $\widetilde{\mathcal{T}}_{\mathrm{Q}}$; Deligne conjectures [16, Conj. 2.10] that $\alpha_{\mathbb{C}}$ itself descends. This would certainly be true if $\alpha_{\mathbb{C}}$ were induced 
by an algebraic correspondence on $\mathcal{Z}_{\mathrm{Q}} \times_{\widetilde{\mathcal{T}}_{\mathrm{Q}}} \mathcal{P}_{\mathrm{Q}}$, as predicted by the Hodge conjecture.

Deligne further gives several equivalent formulations of this conjecture [16. p. 247]. The isomorphism $\beta$ takes the cup product to some multiple $\mu(\beta) \in \mathbb{Q}^{\times}$of the polarization form. This multiple is necessarily constant on all of $\widetilde{\mathcal{T}}_{\mathbb{Q}}$, and the class of $\mu(\beta)$ in $\mathbb{Q}^{\times} /\left(\mathbb{Q}^{\times}\right)^{2}$ is independent of the actual choice of (Q-multiple of) $\beta$. The isomorphism $\alpha_{\mathbb{C}}$ descends to an isomorphism of vector bundles on $\widetilde{\mathcal{T}}_{\mathrm{Q}}$ if and only if $\mu(\beta)$ is a square.

Since $\mu(\beta)$ is constant on $\widetilde{\mathcal{T}}_{\mathrm{Q}}$, to prove (b) it now suffices to prove the Hodge conjecture for a single member of the family $\mathcal{Z}_{\mathrm{Q}} \times \widetilde{\mathcal{T}}_{\mathrm{Q}} \mathcal{P}_{\mathrm{Q}} \rightarrow \widetilde{\mathcal{T}}_{\mathrm{Q}}$. Let $s \in \widetilde{\mathcal{T}}_{\mathbb{Q}}$ represent the Fermat cubic threefold, and let $X_{0}$ be the elliptic curve with complex multiplication by $\mathbb{Z}\left[\zeta_{3}\right]$. The intermediate Jacobian $\mathcal{P}_{S}$ is isogenous to $X_{0}^{\oplus 5}\left([4,11.9]\right.$, [12]). Consequently, $\mathcal{Z}_{s} \times \mathcal{P}_{s}$ is dominated by a product of Fermat hypersurfaces of (prime) degree three, and thus satisfies the Hodge conjecture [43, Thm. IV].

Part (c) follows immediately from (b); after tensoring coefficients with $\mathbb{Q}_{p}$, the crystalline cohomology of a smooth proper family $Y \rightarrow S / \mathbb{F}_{p}$, thought of as a module with connection, may be computed using the de Rham cohomology of a smooth proper lift to characteristic zero [40, Thm. 3.10].

3.4. Prym schemes. Theorem 3.4 proceeds through a Prym construction. It is presumably well-known that one can associate a principally polarized abelian scheme to any étale double cover of relative curves over a base on which 2 is invertible. For want of a suitable reference, details are provided here.

Let $X \rightarrow S$ be an abelian scheme over a connected base, with dual abelian scheme $\hat{X}$. A line bundle $\mathcal{L}$ on $X$ defines a morphism $\phi_{\mathcal{L}}: X \rightarrow \hat{X}$. A polarization of $X$ is an isogeny $\lambda: X \rightarrow \hat{X}$ which, étale-locally on $S$, is of the form $\phi_{\mathcal{L}}$ for some ample $\mathcal{L}$.

Now suppose that $Y$ is a sub-abelian scheme of $X$, with inclusion map $\iota: Y \hookrightarrow X$. A polarization $\lambda$ on $X$ induces a polarization $\lambda_{Y}$ of $Y$. As a map of abelian schemes, it is given by $Y \stackrel{\iota}{\rightarrow} X \stackrel{\lambda}{\rightarrow} \hat{X} \stackrel{\hat{\iota}}{\rightarrow} \hat{Y}$. If $\lambda=\phi_{\mathcal{L}}$ for some ample line bundle $\mathcal{L}$, then $\iota^{*} \mathcal{L}$ is ample on $Y$ and $\lambda_{Y}=\phi_{\iota^{*}} \mathcal{L}$. Since $\lambda_{Y}$ is a surjective map of smooth group schemes, it is flat [34, Lem. 6.12]. In particular, $\operatorname{ker} \lambda_{Y}$ is a finite flat group scheme. The exponent of $Y$ (as a subabelian scheme of the polarized abelian scheme $X)$ is the smallest natural number $e=e(Y \hookrightarrow X, \lambda)$ which annihilates $\operatorname{ker} \lambda_{Y}$.

Lemma 3.7. Let $\iota: Y \hookrightarrow X \rightarrow S$ be an inclusion of abelian schemes over a connected base. Let $\lambda$ be a principal polarization, and suppose that $e=e(Y \hookrightarrow$ $X, \lambda)$ is invertible on $S$. Then there is a complement for $Y$ inside $X$.

Proof. Let $[e]_{Y}$ denote the multiplication-by-e map on $Y$. Since ker $\lambda_{Y} \subseteq$ $\operatorname{ker}[e]_{Y}$, there exists a morphism $\mu_{Y}: \hat{Y} \rightarrow Y$ such that $\mu_{Y} \circ \lambda_{Y}=[e]_{Y}$. 
Consider the norm endomorphism $N_{Y} \in \operatorname{End}(X)$ given by

$$
X \stackrel{\lambda}{\longrightarrow} \hat{X} \stackrel{\hat{\imath}}{\longrightarrow} \hat{Y} \stackrel{\mu_{Y}}{\longrightarrow} Y \stackrel{\iota}{\longrightarrow} X .
$$

The image $N_{X}(X)$ is $Y$. Moreover, the argument of [10, p. 125] shows that $\left.N_{X}\right|_{Y}=[e]_{Y}$. (While the cited result is only claimed for complex abelian varieties, the key calculation [10, Lem. 5.3.1] relies only on the symmetry of the polarization morphism $\lambda$.)

Consider the morphism $M_{Y}:=\mu_{Y} \circ \hat{\imath} \circ \lambda: X \rightarrow Y$ of abelian schemes over $S$. We will show that $M_{Y}$ is smooth. Since $M_{Y}$ is a surjective morphism of smooth group schemes, it is flat. Consequently, in order to show $M_{Y}$ is smooth, it suffices to show that for each point $y \in Y$, the morphism $X_{y} \rightarrow \operatorname{Spec} \kappa(y)$ is smooth, where $\kappa(y)$ is the residue field at $y$ [22. $\mathrm{IV}_{4}$ 17.5.1]. Thus, it suffices to prove the claim in the special case where $S=\operatorname{Spec} K$ is the spectrum of a field in which $e$ is invertible. Because $M_{Y}$ is a homomorphism of group schemes over a field, it suffices to verify its smoothness at a single point [18, VI.1.3]. The respective images $\mathbf{0}_{X}$ and $\mathbf{0}_{Y}$ of the identity sections of $X$ and $Y$ are each, as $S$-schemes, isomorphic to Spec $K$ itself. Therefore, in order to show $M_{Y}$ is smooth, it suffices to show that the induced map on tangent spaces $T_{X}\left(\mathbf{0}_{X}\right) \rightarrow T_{Y}\left(\mathbf{0}_{Y}\right)$ is surjective [22. $\mathrm{IV}_{4}$ 17.11.1]. This last claim is now obvious; because $e$ is invertible in $K$, the differential $[e]_{*}: T_{Y}\left(\mathbf{0}_{Y}\right) \rightarrow T_{Y}\left(\mathbf{0}_{Y}\right)$ is already surjective.

In particular, $M_{Y}: X \rightarrow Y$ is separable, and thus its kernel $Z:=X \times{ }_{Y} \mathbf{0}_{Y}$ is a reduced group scheme over $S$. Its connected component of identity is the sought-for abelian scheme.

Lemma 3.8. Let $S$ be a scheme on which 2 is invertible, and let $\widetilde{C} \rightarrow C / S$ be an étale double cover of smooth proper relative curves over $S$. Then there is a principally polarized complement for $\operatorname{Pic}^{0}(C)$ inside $\operatorname{Pic}^{0}(\widetilde{C})$.

Proof. The relative Picard scheme $\operatorname{Pic}^{0}(\widetilde{C})$ is an abelian scheme over $S$ with canonical principal polarization $\lambda$. The cover of curves yields a canonical inclusion $\operatorname{Pic}^{0}(C) \hookrightarrow \operatorname{Pic}^{0}(\widetilde{C})$, and the exponent of $\operatorname{Pic}^{0}(C)$ is 2. (The exponent may be computed at any (geometric) point of each component of $S$, in which case the result is classical [32. Cor. 1].) By Lemma 3.7, there exists a complement $Z$ to $\operatorname{Pic}^{0}(C)$ inside $\operatorname{Pic}^{0}(\widetilde{C})$. Moreover, the polarization on $Z$ induced by $\lambda$ is twice a principal polarization [32, Cor. 2].

\section{CUBiC SURFACES}

We now resume working in the category of schemes over $\mathcal{O}_{E}[1 / 6]$. As noted in the introduction, to a cubic surface one can associate a cubic threefold. By composing this morphism with $\widetilde{\mathcal{O}}: \widetilde{\mathcal{T}} \rightarrow \mathcal{A}_{5}$ (Corollary 3.5), one obtains a morphism $\widetilde{\mathcal{S}} \rightarrow \mathcal{A}_{5}$. On one hand, the abelian fivefolds thus obtained have an action by $\mathbb{Z}\left[\zeta_{3}\right]$, and the image of this morphism is contained in $\mathcal{M}$. On the other hand, the main result of the present section is that this 
morphism actually descends to a morphism of stacks $\mathcal{S} \rightarrow \mathcal{M}$ (Proposition 4.5) which is injective on points. This is accomplished by introducing rigidifying structures on both sides; a choice of marking of the 27 lines on a cubic surface is tantamount to a level $\left(1-\zeta_{3}\right)$-structure on the associated abelian variety.

4.1. Action of $\mathbb{Z}\left[\zeta_{3}\right]$ on $P(Y)$. Consider the morphism $\widetilde{\phi}: \widetilde{S} \rightarrow \widetilde{\mathcal{T}}$ which sends a cubic form in four variables $f\left(X_{0}, X_{1}, X_{2}, X_{3}\right)$ to the cubic form $X_{4}^{3}-$ $f\left(X_{0}, X_{1}, X_{2}, X_{3}\right)$ in five variables. (This construction is introduced in [4, Sec. 2.1], albeit over $\mathbb{C}$.) Let $j: \mathrm{SL}_{4} \rightarrow \mathrm{SL}_{5}$ be the inclusion $A \mapsto \operatorname{diag}(A, 1)$. Then the diagram

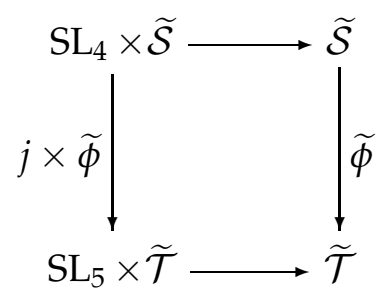

commutes, and $\widetilde{\phi}$ descends to a morphism of stacks

$$
\mathcal{S} \stackrel{\phi}{\longrightarrow} \mathcal{T} \text {. }
$$

This morphism sends a cubic surface $Y$ over an affine scheme Spec $A$ to the cyclic degree-three cover $Z=F(Y)$ of $\mathbb{P}_{A}^{3}$ which is ramified over $Y$. (In the general case of a cubic surface $Y$ inside a projective $S$-bundle $\mathbb{P} V, F(Y)$ is defined by glueing on the base.) We will typically denote this cover by $\pi_{Y}: Z \rightarrow \mathbb{P}^{3}$. Note that, by construction, $\operatorname{Aut}\left(F(Y) / \mathbb{P}^{3}\right) \cong \mathbb{Z} / 3$, say with generator $\gamma$.

Moreover, suppose $f$ is a strictly stable form; it has at least one singularity, and each such looks locally like $x_{1}^{2}+x_{2}^{2}+x_{3}^{2}=0$. Then each singularity of $X_{4}^{3}-f$ looks locally like $x_{4}^{3}-\left(x_{1}^{2}+x_{2}^{2}+x_{3}^{2}\right)=0$, and in particular $X_{4}^{3}-f$ is strictly stable. Consequently, $\widetilde{\phi}$ and $\phi$ extend to morphisms $\widetilde{\mathcal{S}}_{\text {st }} \rightarrow \widetilde{\mathcal{T}}_{\text {st }}$ and $\mathcal{S}_{\text {st }} \rightarrow \mathcal{T}_{\text {st }}$, respectively.

Lemma 4.1. Suppose $S$ is a normal Noetherian scheme and $Y \in \mathcal{S}(S)$. Then $P(F(Y))$ admits multiplication by $\mathbb{Z}\left[\zeta_{3}\right]$.

Proof. Let $X / S$ be any abelian scheme, and let $U \subset S$ be a nonempty open subscheme. Since endomorphisms of $\left.X\right|_{U}$ extend to $X$, it suffices to prove the result when $S=\operatorname{Spec} K$ is the spectrum of a field. Let $Z=F(Y)$, and let $\pi_{Y}: Z \rightarrow \mathbb{P}^{3}$ be the cyclic cubic cover of $\mathbb{P}^{3}$ with branch locus $Y$.

The automorphism $\gamma$ induces an automorphism $\gamma^{*}$ of $A^{2}\left(Z_{\bar{K}}\right)$. Moreover, $\gamma^{*}$ acts nontrivially, for otherwise $\pi_{Y}^{*}: A^{2}\left(\mathbb{P}_{\bar{K}}^{3}\right) \rightarrow A^{2}\left(Z_{\bar{K}}\right)$ would be an isomorphism. The universal property of $P\left(Z_{\bar{K}}\right)$, applied to $\alpha \circ \gamma^{*}$ : $A^{2}(Z) \rightarrow P\left(Z_{\bar{K}}\right)$, shows that $\gamma$ induces a nontrivial automorphism of $P\left(Z_{\bar{K}}\right)$ of order three. By descent, $\gamma$ induces an automorphism of $P(Z)$, and $\mathbb{Z}\left[\zeta_{3}\right] \subseteq$ $\operatorname{End}_{K}(Z)$. 
It remains to check that $1 \in \mathbb{Z}\left[\zeta_{3}\right]$ acts as $[1]_{P(Z)} \in \operatorname{End}(P(Z))$. Any point in $\widetilde{\mathcal{T}}$ of positive characteristic is the specialization of a point in characteristic zero. Since the characteristic polynomial of an endomorphism of an abelian variety is constant in families, it suffices to verify that 1 acts as $[1]_{P(Z)}$ when $K$ has characteristic zero. This claim follows from, e.g., Proposition 3.6 and [4, 2.2].

Remark 4.2. For a suitably generic cubic surface $Y$ over an algebraically closed field $k$, it is possible to visualize the action of $\gamma$, as follows. If $Y$ is generic, then if a plane in $\mathbb{P}^{3}$ intersects $Y$ in two lines, then it does so in three distinct lines. In particular, no plane in $\mathbb{P}^{3}$ tangent to $Y$ is tangent along an entire line of $Y$. So, let $L_{0} \subset Y$ be one of the 27 lines, and let $L=\pi_{Y}^{-1}\left(L_{0}\right) \subset Z$. Since $\pi_{Y}$ is ramified over $Y, L$ is a line inside $Z$, and in fact $L \in F_{Z}^{\text {good }}(k)$. Moreover, $\gamma$ acts on $F$ (by pullback) and fixes $L$ (since $L$ is supported in the ramification locus of $\pi_{Y}$ ). Then $\widetilde{\Delta}_{L}$ and $\Delta_{L}$ are each stable under the action of $\gamma$, and $\mathbb{Z}\left[\zeta_{3}\right]$ acts on each of $\operatorname{Pic}^{0}\left(\widetilde{\Delta}_{L}\right)$ and $\operatorname{Pic}^{0}\left(\Delta_{L}\right)$. The map $\widetilde{\Delta}_{L} \rightarrow \Delta_{L}$ is $\langle\gamma\rangle$-equivariant, and $\mathbb{Z}\left[\zeta_{3}\right]$ acts on the Prym variety $P\left(\widetilde{\Delta}_{L} / \Delta_{L}\right)$.

Lemma 4.3. If $S$ is a normal Noetherian scheme and if $Y \in \mathcal{S}(S)$, then the signature of $P(F(Y))$ is $(4,1)$.

Proof. Since the signature of an $\mathcal{O}_{E}$ action is constant in families (on which the discriminant of $\mathcal{O}_{E}$ is invertible), and since $\mathcal{S}$ is irreducible, it suffices to verify the claim at a single point. A direct Hodge-theoretic calculation [4, Lem. 2.6] shows that the Prym variety associated to any complex cubic surface has signature $(4,1)$.

4.2. Markings and level structure. Suppose that $k$ is algebraically closed and $Y \in \mathcal{S}(k)$, and as usual let $\pi_{Y}: Z=F(Y) \rightarrow \mathbb{P}^{3}$ be the cyclic cubic cover of $\mathbb{P}^{3}$ ramified along $Y$. If $L_{1}$ and $L_{2}$ are lines in $Y$, then the cycle $\left[L_{1}\right]-\left[L_{2}\right]$ is algebraically trivial in $\mathbb{P}^{3}$, and thus $\pi_{\gamma}^{*}\left(\left[L_{1}\right]-\left[L_{2}\right]\right) \in A^{2}(Z)$. Let $\widetilde{L}_{i}=\pi_{Y}^{-1}\left(L_{i}\right)$ for $i \in\{1,2\}$. Then $\pi_{Y}^{*}\left[L_{i}\right]=3\left[\widetilde{L}_{i}\right]$. Since $A^{2}(Z)$ is a divisible group [7, Lem. 0.1.1], $\left[\widetilde{L}_{1}\right]-\left[\widetilde{L}_{2}\right] \in A^{2}(Z)$. Moreover, since each $L_{i}$ is supported in the ramification locus of $\pi_{Y}, \widetilde{L}_{1}$ and $\widetilde{L}_{2}$ are fixed by $\gamma^{*}$. By functoriality of the Prym construction, $\alpha\left(\left[\widetilde{L}_{1}\right]-\left[\widetilde{L}_{2}\right]\right) \in P(Z)\left[1-\zeta_{3}\right](k)$, the kernel on $P(Z)$ of multiplication by $1-\zeta_{3}$.

More generally, suppose $Y / S$ is a smooth cubic surface over a scheme $S$. If $L_{1}$ and $L_{2}$ are relative lines in $Y$, then the difference $\left[\widetilde{L}_{1}\right]-\left[\widetilde{L}_{2}\right]$ of the classes of their inverse images under $\pi_{Y}$ corresponds to a section of $P(Z)\left[1-\zeta_{3}\right]$.

As in [28, p.755], fix isomorphisms $\mathbb{W} \cong \operatorname{Aut}\left(\Lambda_{0}\right)$ and $\mathbb{W} \cong \mathrm{PO}\left(V_{0}, q_{0}\right)$.

Lemma 4.4. Suppose $(Y, \Psi) \in \mathcal{S}^{\mathrm{m}}(S)$, and let $P=P(F(Y))$. For $1 \leq i \leq 5$, let $v_{i}=\alpha\left(\left[\Psi^{-1}\left(e_{i}\right)\right]-\left[\Psi^{-1}\left(\ell_{i 6}\right)\right]\right) \in P(S)$. Then $\left\{v_{1}, \cdots, v_{5}\right\}$ is an orthonormal basis for $P\left[1-\zeta_{3}\right](S)$. 
Proof. Since $(Y, \Psi)$ is the pullback of the universal marked cubic surface over $\mathcal{S}^{\mathrm{m}}$, and since $\mathcal{S}^{\mathrm{m}}$ is itself a normal Noetherian quasiprojective scheme, we may assume that $S$ is normal Noetherian, and even irreducible. Since 3 is invertible on $S$, the group scheme $P\left[1-\zeta_{3}\right]$ is étale over $S$. Therefore, it suffices to show there is some point $s \in S$ such that $\left\{v_{1, s}, \cdots, v_{5, s}\right\}$ is an orthonormal basis for $P\left[1-\zeta_{3}\right]_{S}(s)$. Since $\mathcal{S} \rightarrow \mathcal{O}_{E}[1 / 6]$ is flat, possibly after replacing $S$ with a lift to characteristic zero, we may assume that $S$ has a point whose residue field has characteristic zero. The desired result is then [28, Prop. 5.5].

Proposition 4.5. $\quad$ (a) There is a $\mathbb{W}$-equivariant radicial morphism

$$
\tau^{\mathrm{m}}: \mathcal{S}^{\mathrm{m}} \longrightarrow \mathcal{M}^{\left(1-\zeta_{3}\right)}
$$

of schemes over $\mathcal{O}_{E}[1 / 6]$.

(b) There is a morphism

$$
\tau: \mathcal{S} \longrightarrow \mathcal{M}
$$

of stacks over $\mathcal{O}_{E}[1 / 6]$ which is injective on points and specializes to the complex period map of [4].

Proof. The essential content is already present in Lemma 4.4. If $S$ is a normal Noetherian scheme and $(Y, \Psi)$ is a marked cubic surface over $S$, then the image of the moduli point of $(Y, \Psi)$ is that of the abelian scheme $P(F(Y))$ with its canonical polarization and $\mathcal{O}_{E}$-action, with level structure $\Phi: v_{i} \mapsto$ $\alpha\left(\left[\Psi^{-1}\left(e_{i}\right)\right]-\left[\Psi^{-1}\left(\ell_{i 6}\right)\right]\right)$. By applying this construction to $\mathcal{S}^{\mathrm{m}}$ itself we obtain a $\mathcal{S}^{\mathrm{m}}$-valued point of $\mathcal{M}^{\left(1-\zeta_{3}\right)}$, i.e., a morphism $\mathcal{S}^{\mathrm{m}} \rightarrow \mathcal{M}^{\left(1-\zeta_{3}\right)}$. The $\mathbb{W}$-equivariance is worked out in detail by Matsumoto and Terasoma; see [28, Sec. 3.2, Prop. 5.5]. Beauville's Torelli-type theorem [8, Cor. on p.205] shows that this morphism is injective on geometric points. Therefore, $\tau^{\mathrm{m}}$ is radicial, i.e., injective on $K$-points for any field $K$ admitting a map $\mathcal{O}_{E}[1 / 6] \rightarrow K$. This proves (a).

For (b), use part (a) and the identifications $\mathcal{S}=\mathcal{S}^{\mathrm{m}} / \mathbb{W}$ and $\mathcal{M}=\mathcal{M}^{\left(1-\zeta_{3}\right)} / \mathbb{W}$. The fact that $\tau_{\mathrm{C}}$ specializes to the period map of [4] follows from the known isomorphism (Section 3.1) between the Prym $P(Z)$ and the intermediate Jacobian $J(Z)$ of a smooth complex cubic threefold. 
Thus, we have constructed morphisms $\widetilde{\omega}$ and $\tau$ as follows:

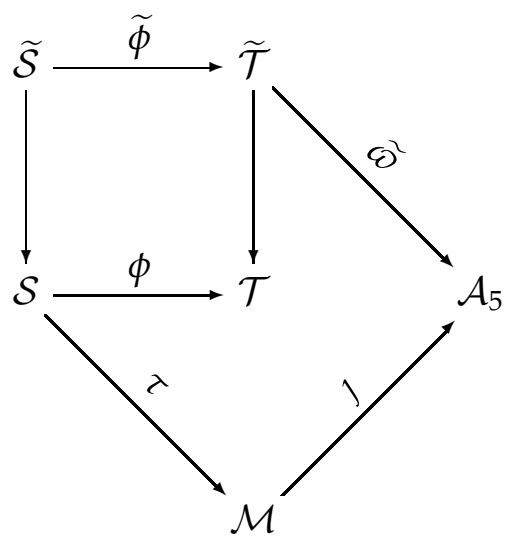

Since $\mathcal{S}$ and $\mathcal{M}$ have irreducible four-dimensional fibers over $\operatorname{Spec} \mathcal{O}_{E}[1 / 6]$, and since $\tau$ is radicial, it follows that the image of $\tau$ is open in $\mathcal{M}$. Describing this image precisely requires a consideration of the compactification of $\tau$.

\section{Characterization of The image}

The goal of the present section is to extend $\tau$ to a homeomorphism $\mathcal{S}_{\mathrm{st}} \rightarrow$ $\mathcal{M}$ (Theorem 5.7), and thus give an arithmetic version of many of the complex analytic facts established in [4]. In particular, the complement of $\tau(\mathcal{S})$ in $\mathcal{M}$ is an irreducible horizontal divisor which corresponds to cubic surfaces which are stable but not smooth.

Lemma 5.1. The complement $\widetilde{\mathcal{S}}_{\text {st }} \backslash \widetilde{\mathcal{S}}$ is an irreducible horizontal divisor in $\widetilde{\mathcal{S}}_{\text {st. }}$.

Proof. We need to show that if $s \in \operatorname{Spec} \mathcal{O}_{E}[1 / 6]$, then $\left(\widetilde{\mathcal{S}}_{\text {st }} \backslash \widetilde{\mathcal{S}}\right)_{s}$ is an irreducible divisor in $\widetilde{\mathcal{S}}_{\mathrm{st}, \mathrm{s}}$. This is [9, Prop. 6.7]; while the result is claimed only for $\left(\widetilde{\mathcal{S}}_{\text {st }} \backslash \widetilde{\mathcal{S}}\right)_{\mathrm{C}}$, the argument given there is valid over an arbitrary base field.

Let $\widetilde{\tau}$ be the composition $\widetilde{\mathcal{S}} \rightarrow \mathcal{S} \stackrel{\tau}{\rightarrow} \mathcal{M}$.

Lemma 5.2. The Torelli map $\widetilde{\tau}: \widetilde{\mathcal{S}} \rightarrow \mathcal{M}$ extends to $\widetilde{\tau}: \widetilde{\mathcal{S}}_{\mathrm{st}} \rightarrow \mathcal{M}$.

Proof. The existence of $\widetilde{\mathcal{S}}_{\text {st, } \mathrm{C}} \rightarrow \mathcal{M}_{\mathbb{C}}$ is [4, Thm. 3.17]. By descent (e.g., [25, 15.8]) one obtains $\widetilde{\mathcal{S}}_{\mathrm{st}, E} \rightarrow \mathcal{M}_{E}$.

Now let $\mathfrak{p} \subset \mathcal{O}_{E}[1 / 6]$ be a nonzero prime ideal, and let $\mathcal{O}_{E,(\mathfrak{p})}$ be the localization at $\mathfrak{p}$; it is an unramified discrete valuation ring of mixed characteristic. Let $[\mathfrak{p}]$ be the closed point of $\operatorname{Spec} \mathcal{O}_{E,(\mathfrak{p})}$. Finally, let $S=\widetilde{\mathcal{S}}_{\text {st }} \times$ Spec $\mathcal{O}_{E,(\mathfrak{p})}$, and let $T=\left(\widetilde{\mathcal{S}}_{\text {st }} \backslash \widetilde{\mathcal{S}}\right)_{[\mathfrak{p}]}$. Then $S$ is smooth, and $T$ is a closed subscheme of codimension two supported over the closed point of $\operatorname{Spec} \mathcal{O}_{E,(\mathfrak{p})}$. A result of Faltings [31, Lemma 3.6] shows that the abelian scheme over 
$S \backslash T$ extends uniquely to one over all of $S$. Since the $\mathcal{O}_{E}$-action also extends to this abelian scheme, and the signature is constant, we obtain a morphism $S \rightarrow \mathcal{M}$. Glueing yields the desired extension $\widetilde{\mathcal{S}}_{\text {st }} \rightarrow \mathcal{M}$.

Lemma 5.3. The marked Torelli map $\tau^{\mathrm{m}}: \mathcal{S}^{\mathrm{m}} \rightarrow \mathcal{M}^{\left(1-\zeta_{3}\right)}$ extends to $\mathcal{S}_{\mathrm{st}}^{\mathrm{m}} \rightarrow$ $\mathcal{M}^{\left(1-\zeta_{3}\right)}$ and to $\mathcal{S}_{\mathrm{ss}}^{\mathrm{m}} \rightarrow \overline{\mathcal{M}}^{\left(1-\zeta_{3}\right)}$. The Torelli map $\tau: \mathcal{S} \rightarrow \mathcal{M}$ extends to $\mathcal{S}_{\mathrm{st}} \rightarrow \mathcal{M}$ and to $\mathcal{S}_{\mathrm{ss}} \rightarrow \overline{\mathcal{M}}$.

Proof. As in Section 2.1, let $\underline{S}_{s t}$ be the underlying coarse moduli scheme of $\mathcal{S}_{\text {st }}$, and similarly define $\underline{M}$. (Recall that $\mathcal{S}_{\text {st }}^{\mathrm{m}}$ is itself already a scheme.) Since the morphism $\widetilde{\tau}: \widetilde{\mathcal{S}}_{\text {st }} \rightarrow \mathcal{M}$ of Lemma 5.2 is constant on the fibers of $\widetilde{\mathcal{S}}_{\mathrm{st}} \rightarrow \mathcal{S}_{\mathrm{st}}$, we have a map of normal quasiprojective schemes $\underline{\tau}: \underline{S}_{\mathrm{st}} \rightarrow$ $\underline{M}$. Via the projection $\mathcal{S}_{\mathrm{st}}^{\mathrm{m}} \rightarrow \underline{S}_{\mathrm{st}}$, we obtain a dominant morphism $\psi$ : $\overline{\mathcal{S}_{\mathrm{st}}^{\mathrm{m}}} \rightarrow \underline{M}$ which, as a rational map, factors through $\mathcal{M}^{\left(1-\zeta_{3}\right)}$. Therefore, as a morphism of schemes, $\psi$ factors through the normalization of $M$ in $\mathcal{M}^{\left(1-\zeta_{3}\right)}$; since $\mathcal{M}^{\left(1-\zeta_{3}\right)}$ is already a normal scheme, $\psi$ factors through $\tau^{\mathrm{m}}$ : $\mathcal{S}_{\mathrm{st}}^{\mathrm{m}} \rightarrow \mathcal{M}^{\left(1-\zeta_{3}\right)}$.

This proves the first part of the claim. The extension of $\tau^{\mathrm{m}}$ to $\mathcal{S}_{\mathrm{ss}}$ follows from [22, $\mathrm{IV}_{4}$.20.4.12]; by choosing an affine neighborhood of each cusp, we have a rational map defined on an open subset of a normal affine scheme whose complement has codimension greater than one.

Finally, the extension of $\tau$ follows from the $\mathbb{W}$-equivariance of $\tau^{\mathrm{m}}$.

Ultimately, it will turn out that $\tau$ is injective on points. As a preliminary step, we will show that $\tau$ preserves the stratification $\mathcal{S} \subsetneq \mathcal{S}_{\text {st }} \subsetneq S_{\text {ss }}$.

Lemma 5.4. We have $\tau(\mathcal{S}) \cap \tau\left(\mathcal{S}_{\text {st }} \backslash \mathcal{S}\right)=\varnothing$ and $\tau\left(\mathcal{S}_{\text {st }}\right) \cap \tau\left(\mathcal{S}_{\text {ss }} \backslash \mathcal{S}_{\text {st }}\right)=\varnothing$.

Proof. The unique point of $\left(\mathcal{S}_{\mathrm{ss}} \backslash \mathcal{S}_{\mathrm{st}}\right)(\mathbb{C})$ is sent to the unique cusp of $\overline{\mathcal{M}}_{\mathrm{C}}$, and thus the same is true for every geometric fiber of $\mathcal{S}_{\mathrm{ss}} \rightarrow \operatorname{Spec} \mathcal{O}_{E}[1 / 6]$. Since $\tau\left(\mathcal{S}_{\mathrm{st}}\right) \subset \mathcal{M}$, the second claim follows immediately.

The first claim will be achieved using the explicit nature of the Torelli theorem for cubic threefolds [8]. Suppose $Z / k$ is a smooth cubic threefold over an algebraically closed field, with principally polarized Prym variety $(X, \lambda)$. Consider the theta divisor $\Theta \subset X$ associated to $\lambda$. Then $\Theta$ has a unique singularity, which is a triple point; and $Z$ (actually, its affine cone) may be reconstructed as the tangent cone at that singularity.

Moreover, if $k=\mathbb{C}$, a converse is available [13]; a principally polarized abelian fivefold $(X, \lambda)$ is the Prym variety of a smooth cubic threefold if and only if its theta divisor has a unique singularity, which is a singularity of order three.

In fact, the theta divisor of a stable, but not smooth, cubic threefold must be more singular than that of a smooth cubic threefold, as follows.

Let $Y / \mathbb{C}$ be a nodal stable cubic surface. Then $Z=F(Y)$ is a nodal stable cubic threefold. Because $Z$ can be realized as a degeneration of smooth cubic threefolds, the theta divisor $\Theta$ of $(P(Z), \lambda)$ has at least one singular point of order at least three. In fact, either $\Theta$ has a second singular point, or 
its unique singularity has order greater than three; for if not, then by [13], $(P(X), \lambda)$ would also be the Prym of a smooth cubic threefold (still with suitable $\mathbb{Z}\left[\zeta_{3}\right]$-action), which would contradict the known injectivity of $\tau_{\mathbb{C}}$ on $\mathcal{S}_{\mathrm{st}, \mathrm{C}}$ [4, Thm. 3.17].

Similarly, let $Y / k$ be a nodal stable cubic surface over a field of characteristic at least 5 . Let $R$ be a mixed characteristic discrete valuation ring with residue field $k$; let $K=$ Frac $R$ be its fraction field. Lift $Y$ to a cubic surface $\widetilde{Y} / R$ such that $\widetilde{Y}_{K}$ is also nodal stable. We have seen that the theta divisor of the principally polarized abelian variety $P\left(F\left(\widetilde{Y}_{K}\right)\right)$ either has at least two singular points, or has one singularity of order greater than three. The same is necessarily true of $P\left(F\left(\widetilde{Y}_{k}\right)\right)=P(F(Y))$, its specialization.

In summary, the geometry of the theta divisor means that the Prym variety of a nodal stable cubic surface cannot also be the Prym variety of a smooth cubic surface.

Let $\mathcal{N}$ be the image of $\mathcal{S}$ under $\tau$, and let $\mathcal{D}$ be its complement. Then $\mathcal{N}^{(1-\zeta)}:=\mathcal{N} \times \mathcal{M}_{\mathcal{M}} \mathcal{M}^{(1-\zeta)}$ is the image of $\mathcal{S}^{\mathrm{m}}$ under $\tau^{\mathrm{m}}$, with complement $\mathcal{D}^{(1-\zeta)}$.

Lemma 5.5. The marked Torelli morphism maps $\mathcal{S}^{\mathrm{m}}$ isomorphically onto its image $\mathcal{N}^{(1-\zeta)}$.

Proof. Because $\left.\tau^{\mathrm{m}}\right|_{\mathcal{S}^{\mathrm{m}}}$ is radicial (Proposition 4.5) and of finite type, it is quasifinite. Since $\mathcal{S}_{\mathrm{ss}}^{\mathrm{m}}$ is projective, $\tau^{\mathrm{m}}$ is projective. Lemma 5.4 shows that $\mathcal{S}_{\text {ss }}^{\mathrm{m}} \times{ }_{\mathcal{M}^{(1-\zeta)}} \mathcal{N}^{(1-\zeta)}$ is $\mathcal{S}^{\mathrm{m}}$ (and not larger), and so $\left.\tau^{\mathrm{m}}\right|_{\mathcal{S}^{\mathrm{m}}}$ is proper, thus finite.

Now, $\mathcal{N}^{(1-\zeta)}$ is smooth, and in particular its local rings are unique factorization domains. By computing locally on $\mathcal{N}^{(1-\zeta)}$, we see that the finite morphism $\left.\tau^{\mathrm{m}}\right|_{\mathcal{S}^{\mathrm{m}}}$ is necessarily flat [29, Thm. 22.6]. In particular, its degree is constant. Since that degree is known to be one in characteristic zero [4, Thm. 3.17], $\left.\tau^{\mathrm{m}}\right|_{\mathcal{S}^{\mathrm{m}}}$ is an isomorphism onto its image.

Lemma 5.6. The marked Torelli morphism $\tau^{\mathrm{m}}: \mathcal{S}_{\mathrm{ss}}^{\mathrm{m}} \rightarrow \overline{\mathcal{M}}^{\left(1-\zeta_{3}\right)}$ is an isomorphism.

Proof. Each of $\mathcal{S}_{\mathrm{ss}}^{\mathrm{m}} \rightarrow \operatorname{Spec} \mathcal{O}_{E}[1 / 6]$ and $\overline{\mathcal{M}}^{(1-\zeta)} \rightarrow \operatorname{Spec} \mathcal{O}_{E}[1 / 6]$ has fourdimensional geometric fibers. Lemma 5.5 implies that, fiberwise on $\operatorname{Spec} \mathcal{O}_{E}[1 / 6]$, $\tau^{\mathrm{m}}$ is a birational morphism. Moreover, over the generic fiber of $\operatorname{Spec} \mathcal{O}_{E}[1 / 6]$, $\tau^{\mathrm{m}}$ is an isomorphism [4. Thm. 3.17]. In particular, $\tau^{\mathrm{m}}$ is a birational morphism.

Let $\sigma$ be the inverse of $\tau^{\mathrm{m}}$ in the category of rational maps. Suppose $\mathfrak{p} \subset \mathcal{O}_{E}[1 / 6]$ is a nonzero prime ideal. The locus of indeterminacy of $\sigma_{[\mathfrak{p}]}$ has positive codimension in $\overline{\mathcal{M}}_{[\mathfrak{p}]}^{\left(1-\zeta_{3}\right)}$, and thus the locus of indeterminacy of $\sigma$ on $\overline{\mathcal{M}}^{\left(1-\zeta_{3}\right)} \times \operatorname{Spec} \mathcal{O}_{E,(\mathfrak{p})}$ has codimension at least two. Since $\overline{\mathcal{M}}^{\left(1-\zeta_{3}\right)} \times$ Spec $\mathcal{O}_{E,(\mathfrak{p})}$ is normal and $\tau^{\mathrm{m}}$ is proper, $\sigma$ is defined on all of $\overline{\mathcal{M}}^{\left(1-\zeta_{3}\right)}$ and $\tau^{\mathrm{m}}$ is biregular. 
We now come to the arithmetic analogue of the main results of [4]. In [4], Theorem 3.20 states that the stacks $\mathcal{S}_{\text {st,C }}$ and $\mathcal{M}_{\mathrm{C}}$ are isomorphic, and Theorems 3.17 and 8.2 identify the GIT compactification $\mathcal{S}_{\mathrm{ss}, \mathrm{C}}$ with the minimal compactification $\overline{\mathcal{M}}_{\mathrm{C}}$. These assertions are actually specializations of maps of stacks over $\mathbb{Z}\left[\zeta_{3}, 1 / 6\right]$ :

Theorem 5.7. The Torelli morphisms $\tau$ and $\tau^{\mathrm{m}}$ give rise to the commutative diagram of stacks over $\mathbb{Z}\left[\zeta_{3}, 1 / 6\right]$

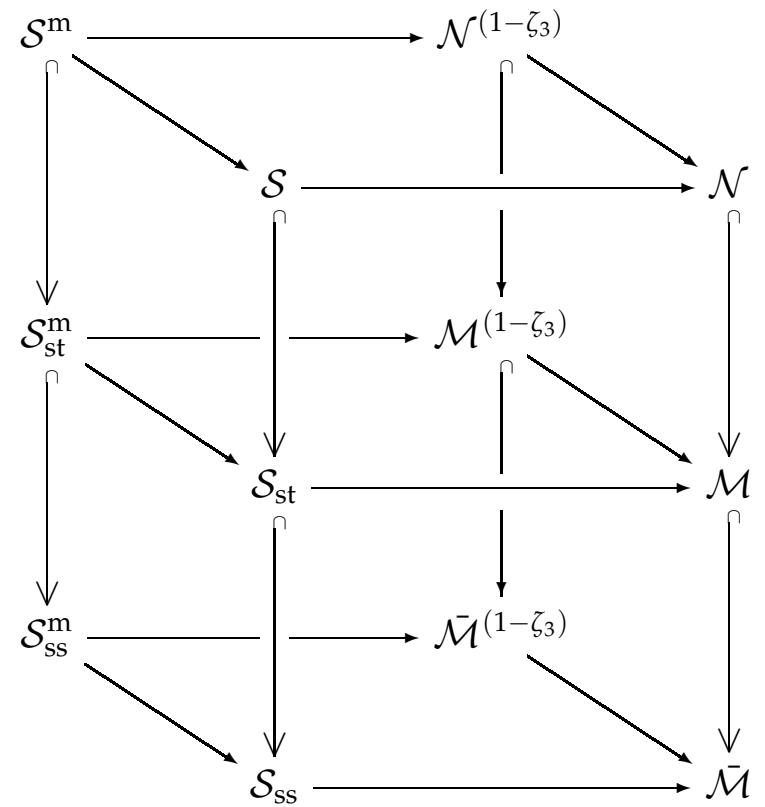

in which all vertical arrows are the natural inclusions; all diagonal arrows are quotients by the action of $\mathbb{W}$; all horizontal arrows are homeomorphisms; the rear horizontal arrows are isomorphisms of schemes; and the top front horizontal arrow is an isomorphism of stacks.

Proof. The assertions for the top (horizontal) square follow from Lemma 5.5 and $\mathbb{W}$-equivariance; those for the bottom, from Lemma 5.6. Every geometric fiber of $\overline{\mathcal{M}} \backslash \mathcal{M}$ consists of a single point, as does each geometric fiber of $\mathcal{S}_{\text {ss }} \backslash \mathcal{S}_{\text {st }}$. Consequently, the image of $\mathcal{S}_{\text {st }}$ under $\tau$ is exactly $\mathcal{M}$, which finishes the proof of the theorem.

Corollary 5.8. Let $\mathcal{D}$ be the complement of $\mathcal{N}$ in $\mathcal{M}$. Then $\mathcal{D}$ is an irreducible horizontal divisor.

In fact, $\mathcal{D}$ is a special cycle in the sense of Kudla and Rapoport [25].

Proof. By Theorem 5.7 and Lemma 5.1, $\mathcal{D}$ is, fiberwise on $\operatorname{Spec} \mathcal{O}_{E}[1 / 6]$, a closed irreducible substack of $\mathcal{M}$. It therefore suffices to identify a closed horizontal irreducible substack of $\mathcal{N}$ which has codimension one in $\mathcal{M}$. The Prym variety associated to a smooth cubic threefold, and in particular to the triple cover of $\mathbb{P}^{3}$ ramified along a given smooth cubic surface, is 
irreducible as a principally polarized abelian variety [8, 15]. Therefore, it suffices to exhibit a horizontal divisor in $\mathcal{M}$ which parametrizes reducible principally polarized abelian varieties with $\mathcal{O}_{E}$-action. Let $\mathcal{M}_{(3,1)}$ be the moduli stack of abelian fourfolds with action by $\mathcal{O}_{E}$ of signature $(3,1)$, and let $\left(X_{0}, \iota_{0}, \lambda_{0}\right) / \mathcal{O}_{E}[1 / 6]$ be the (unique, principally polarized) elliptic curve with action by $\mathcal{O}_{E}$ of signature $(1,0)$. There is a closed immersion of stacks $\mathcal{M}_{(3,1)} \rightarrow \mathcal{M}$; on $S$-points, it is given by $(X, l, \lambda) \mapsto\left(\left(X_{0} \times S\right) \times X, \iota_{0} \times\right.$ $\left.\iota, \lambda_{0} \times \lambda\right)$. Let $\mathcal{D}^{\prime}$ be the image of this morphism. Then every fiber of $\mathcal{D}^{\prime} \rightarrow$ $\mathcal{O}_{E}[1 / 6]$ has dimension 3 , and $\mathcal{D}=\mathcal{D}^{\prime}$ is the sought-for divisor.

The modular interpretation of $\mathcal{D}$ is compatible with the discussion of intermediate Jacobians of strictly stable cubic threefolds in [14]. A strictly stable cubic surface $Y$ has a singularity of type $A_{1}$. We have noted above that the cyclic cubic threefold $F(Y)$ has a singularity of type $A_{2}$. Consequently (at least in characteristic zero), $P(F(Y)$ ) has an elliptic tail [14, Thm. 6.3]. The presence of the $\zeta_{3}$ action means this tail must, in fact, be the elliptic curve $\left(X_{0}, \iota_{0}, \lambda_{0}\right)$ introduced in Corollary 5.8 .

Let $(X, \lambda)$ be a principally polarized abelian variety over $\mathbb{C}$ of dimension five. Recall that Casalaina-Martin and Friedman have shown [13] that $(X, \lambda)$ is the intermediate Jacobian of a smooth cubic threefold if and only if its theta divisor has a unique singularity, and that singularity has order three. Their result implies an analogous result for abelian varieties with $\mathbb{Z}\left[\zeta_{3}\right]$-action, which is valid in all characteristics:

Corollary 5.9. Let $(X, l, \lambda) \in \mathcal{M}(k)$ be a principally polarized abelian fivefold with action by $\mathbb{Z}\left[\zeta_{3}\right]$. Then either the theta divisor of $(X, \lambda)$ has a unique singularity, and that singularity is of order three, in which case $(X, \lambda)=P(F(Y))$ for a smooth cubic surface $Y$; or the theta divisor has a positive-dimensional singular locus, in which case $(X, \lambda)=P(F(Y))$ for a nodal cubic surface $Y$. In each case, $Y$ is determined uniquely by $(X, l, \lambda)$.

Proof. By Theorem 5.7, if $(X, l, \lambda) \in \mathcal{M}(k)$, then there is a unique $Y \in \mathcal{S}_{\text {st }}(k)$ such that $X=P(F(Y))$. On one hand, if $Y$ is smooth, then we have seen in the proof of Lemma 5.4 that the theta divisor of $(X, \lambda)$ has a unique singularity, and that singularity is of order three. On the other hand, if $Y$ is stable but not smooth, then $(X, l, \lambda)$ is the product of an elliptic curve and an abelian fourfold (Corollary 5.8), and thus the theta divisor of $(X, \lambda)$ is singular along a positive-dimensional subvariety.

\section{REFERENCES}

[1] Jeffrey D. Achter, On the abelian fivefolds attached to cubic surfaces, August 2012, arXiv:1208.2974, arXiv:1208.2974.

[2] Daniel Allcock, The moduli space of cubic threefolds, J. Algebraic Geom. 12 (2003), no. 2, 201-223, 10.1090/S1056-3911-02-00313-2.

[3] Daniel Allcock, James A. Carlson, and Domingo Toledo, A complex hyperbolic structure for moduli of cubic surfaces, C. R. Acad. Sci. Paris Sér. I Math. 326 (1998), no. 1, 49-54. 
[4] The complex hyperbolic geometry of the moduli space of cubic surfaces, J. Algebraic Geom. 11 (2002), no. 4, 659-724.

[5] _ The moduli space of cubic threefolds as a ball quotient, Mem. Amer. Math. Soc. 209 (2011), no. 985, xii+70, 10.1090/S0065-9266-10-00591-0.

[6] Allen B. Altman and Steven L. Kleiman, Foundations of the theory of Fano schemes, Compositio Math. 34 (1977), no. 1, 3-47.

[7] Arnaud Beauville, Variétés de Prym et jacobiennes intermédiaires, Ann. Sci. École Norm. Sup. (4) 10 (1977), no. 3, 309-391.

[8] __ Les singularités du diviseur $\Theta$ de la jacobienne intermédiaire de l'hypersurface cubique dans $\mathbf{P}^{4}$, Algebraic threefolds (Varenna, 1981), Lecture Notes in Math., vol. 947, Springer, Berlin, 1982, pp. 190-208.

[9] - Moduli of cubic surfaces and Hodge theory (after Allcock, Carlson, Toledo), Géométries à courbure négative ou nulle, groupes discrets et rigidités, Sémin. Congr., vol. 18, Soc. Math. France, Paris, 2009, pp. 445-466.

[10] Christina Birkenhake and Herbert Lange, Complex abelian varieties, second ed., Grundlehren der Mathematischen Wissenschaften [Fundamental Principles of Mathematical Sciences], vol. 302, Springer-Verlag, Berlin, 2004.

[11] Siegfried Bosch, Werner Lütkebohmert, and Michel Raynaud, Néron models, Ergebnisse der Mathematik und ihrer Grenzgebiete (3) [Results in Mathematics and Related Areas (3)], vol. 21, Springer-Verlag, Berlin, 1990.

[12] James A. Carlson and Domingo Toledo, Cubic surfaces with special periods, October 2011, arXiv:1104.1782, to appear, Proc. AMS.

[13] Sebastian Casalaina-Martin and Robert Friedman, Cubic threefolds and abelian varieties of dimension five, J. Algebraic Geom. 14 (2005), no. 2, 295-326, 10.1090/S1056-3911-0400379-0.

[14] Sebastian Casalaina-Martin and Radu Laza, The moduli space of cubic threefolds via degenerations of the intermediate Jacobian, J. Reine Angew. Math. 633 (2009), 29-65, 10.1515/CRELLE.2009.059.

[15] C. Herbert Clemens and Phillip A. Griffiths, The intermediate Jacobian of the cubic threefold, Ann. of Math. (2) 95 (1972), 281-356.

[16] Pierre Deligne, Les intersections complètes de niveau de Hodge un, Invent. Math. 15 (1972), 237-250.

[17] Pierre Deligne and G. Daniel Mostow, Monodromy of hypergeometric functions and nonlattice integral monodromy, Inst. Hautes Études Sci. Publ. Math. (1986), no. 63, 5-89.

[18] Michel Demazure and Alexander Grothendieck, Schémas en groupes, séminaire de géométrie algébrique du bois marie 1962/64 (sga 3)., Lecture Notes in Mathematics, no. 151, 152, 153, Springer-Verlag, Berlin, 1962/1964.

[19] Igor Dolgachev, Bert van Geemen, and Shigeyuki Kondō, A complex ball uniformization of the moduli space of cubic surfaces via periods of K3 surfaces, J. Reine Angew. Math. $\mathbf{5 8 8}$ (2005), 99-148, 10.1515/crll.2005.2005.588.99.

[20] Igor V. Dolgachev and Shigeyuki Kondō, Moduli of K3 surfaces and complex ball quotients, Arithmetic and geometry around hypergeometric functions, Progr. Math., vol. 260, Birkhäuser, Basel, 2007, 10.1007/978-3-7643-8284-13, pp. 43-100.

[21] Gerd Faltings and Ching-Li Chai, Degeneration of abelian varieties, Springer-Verlag, Berlin, 1990, With an appendix by David Mumford.

[22] Alexander Grothendieck, Éléments de géométrie algébrique. IV. Étude locale des schémas et des morphismes de schémas, Inst. Hautes Études Sci. Publ. Math. (1964-1967), no. 20, 24, $28,32$.

[23] Steven L. Kleiman, The Picard scheme, Fundamental algebraic geometry, Math. Surveys Monogr., vol. 123, Amer. Math. Soc., Providence, RI, 2005, pp. 235-321. 
[24] Shigeyuki Kondō, The moduli space of curves of genus 4 and Deligne-Mostow's complex reflection groups, Algebraic geometry 2000, Azumino (Hotaka), Adv. Stud. Pure Math., vol. 36, Math. Soc. Japan, Tokyo, 2002, pp. 383-400.

[25] Stephen Kudla and Michael Rapoport, Special cycles on unitary Shimura varieties II: global theory, December 2009, arXiv:0912.3758, arXiv:0912.3758.

[26] __ On occult period maps, March 2012, arXiv:1203.1272, arXiv:1203.1272.

[27] Eduard Looijenga and Rogier Swierstra, On period maps that are open embeddings, J. Reine Angew. Math. 617 (2008), 169-192, 10.1515/CRELLE.2008.029.

[28] Keiji Matsumoto and Tomohide Terasoma, Theta constants associated to cubic threefolds, J. Algebraic Geom. 12 (2003), no. 4, 741-775.

[29] Hideyuki Matsumura, Commutative ring theory, second ed., Cambridge Studies in Advanced Mathematics, vol. 8, Cambridge University Press, Cambridge, 1989, Translated from the Japanese by M. Reid.

[30] James S. Milne, Algebraic geometry (v5.20), 2009, Available at www.jmilne.org/math/, pp. 239+vi.

[31] Ben Moonen, Models of Shimura varieties in mixed characteristics, Galois representations in arithmetic algebraic geometry (Durham, 1996), Cambridge Univ. Press, Cambridge, 1998, pp. 267-350.

[32] David Mumford, Prym varieties. I, Contributions to analysis (a collection of papers dedicated to Lipman Bers), Academic Press, New York, 1974, pp. 325-350.

[33] __ Stability of projective varieties, Enseignement Math. (2) 23 (1977), no. 1-2, 39-110.

[34] David Mumford, John Fogarty, and Frances Kirwan, Geometric invariant theory, Ergebnisse der Mathematik und ihrer Grenzgebiete (2) [Results in Mathematics and Related Areas (2)], vol. 34, Springer-Verlag, Berlin, 1994.

[35] _ Geometric invariant theory, Ergebnisse der Mathematik und ihrer Grenzgebiete (2) [Results in Mathematics and Related Areas (2)], vol. 34, Springer-Verlag, Berlin, 1994.

[36] Jacob P. Murre, Algebraic equivalence modulo rational equivalence on a cubic threefold, Compositio Math. 25 (1972), 161-206.

[37] _ Reduction of the proof of the non-rationality of a non-singular cubic threefold to a result of Mumford, Compositio Math. 27 (1973), 63-82.

[38] __ Some results on cubic threefolds, Classification of algebraic varieties and compact complex manifolds, Springer, Berlin, 1974, pp. 140-160. Lecture Notes in Math., Vol. 412.

[39] Isao Naruki, Cross ratio variety as a moduli space of cubic surfaces, Proc. London Math. Soc. (3) 45 (1982), no. 1, 1-30, 10.1112/plms/s3-45.1.1, With an appendix by Eduard Looijenga.

[40] Arthur Ogus, F-isocrystals and de Rham cohomology. II. Convergent isocrystals, Duke Math. J. 51 (1984), no. 4, 765-850, 10.1215/S0012-7094-84-05136-6.

[41] M. Rapoport, Complément à l'article de P. Deligne "La conjecture de Weil pour les surfaces K3", Invent. Math. 15 (1972), 227-236.

[42] C. S. Seshadri, Quotient spaces modulo reductive algebraic groups, Ann. of Math. (2) 95 (1972), 511-556; errata, ibid. (2) 96 (1972), 599.

[43] Tetsuji Shioda, The Hodge conjecture for Fermat varieties, Math. Ann. 245 (1979), no. 2, 175-184, 10.1007/BF01428804.

[44] Alice Silverberg, Fields of definition for homomorphisms of abelian varieties, J. Pure Appl. Algebra 77 (1992), no. 3, 253-262.

E-mail address: j.achter@colostate.edu

Department of Mathematics, Colorado State University, Fort Collins, CO 80523

URL: http://www . math. colostate. edu/ ${ }^{\sim}$ achter 